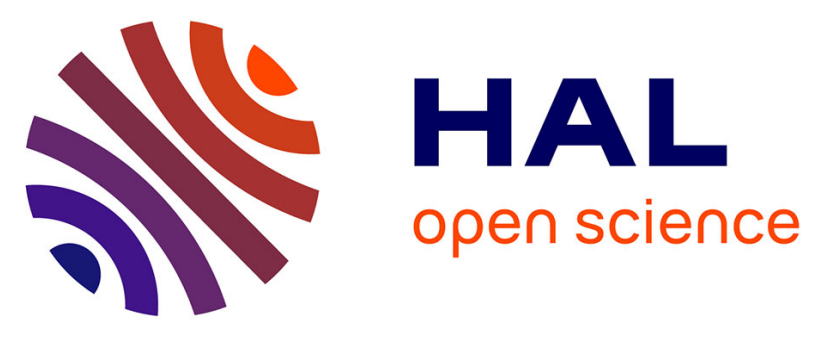

\title{
Etude morphosédimentaire d'un remplissage endokarstique de la grotte du bois du clos (Charente, France) : contraintes géométriques et dynamiques hydrologiques
}

Grégory Dandurand, Guillaume Devès, Richard Maire, Richard Ortega, Dominique Genty, Bassam Ghaleb

\section{To cite this version:}

Grégory Dandurand, Guillaume Devès, Richard Maire, Richard Ortega, Dominique Genty, et al.. Etude morphosédimentaire d'un remplissage endokarstique de la grotte du bois du clos (Charente, France) : contraintes géométriques et dynamiques hydrologiques. Quaternaire, 2011, 22 (4), pp.285306. 10.4000/quaternaire.6019 . hal-02269051

\section{HAL Id: hal-02269051 https://hal.science/hal-02269051}

Submitted on 19 Oct 2020

HAL is a multi-disciplinary open access archive for the deposit and dissemination of scientific research documents, whether they are published or not. The documents may come from teaching and research institutions in France or abroad, or from public or private research centers.
L'archive ouverte pluridisciplinaire HAL, est destinée au dépôt et à la diffusion de documents scientifiques de niveau recherche, publiés ou non, émanant des établissements d'enseignement et de recherche français ou étrangers, des laboratoires publics ou privés. 


\title{
ÉTUDE MORPHOSÉDIMENTAIRE D'UN REMPLISSAGE ENDOKARSTIQUE DE LA GROTTE DU BOIS DU CLOS (CHARENTE, FRANCE) : CONTRAINTES GÉOMÉTRIQUES ET DYNAMIQUES HYDROLOGIQUES
}

\author{
Grégory DANDURAND ${ }^{1}$, Guillaume DEVÈS ${ }^{2}$, Richard MAIRE ${ }^{1}$, Richard ORTEGA $^{2}$, \\ Dominique GENTY ${ }^{3} \&$ Bassam GHALEB ${ }^{4}$
}

\begin{abstract}
RÉSUMÉ
Une coupe dans un remplissage détritique remarquable de plus de $5 \mathrm{~m}$ de hauteur, situé dans la grotte du Bois du Clos (karst de La Rochefoucauld, Charente) a permis une analyse morphosédimentaire d'une séquence discontinue et complexe. Trois grandes phases successives d'ennoyage/dénoyage de la cavité ont été mises en évidence par la combinaison d'une analyse granulométrique, des diagrammes de Passega, d'une analyse minéralogique des sables et des argiles et d'une analyse géochimique. Malgré l'absence de jalons chronologiques à l'intérieur de la séquence sédimentaire, une chronostratigraphie et un calage chronologique sont proposés grâce à une stalagmite qui scelle le remplissage et datée du stade isotopique marin 3. Les résultats sont corrélés avec des épisodes mis en évidence dans des sites voisins. Ils montrent dans notre cas le paléofonctionnement d'un système karstique d'abord ouvert et largement influencé par la fluctuation d'une grande nappe karstique (la Touvre) et par des écoulements proches (paléopertes), mais qui progressivement va se fermer. La question de l'interprétation paléoclimatique et paléoenvironnementale des sédiments est discutée. Les notions de piège karstique et de lacune sédimentaire sont développées. Les effets de site sur l'enregistrement sédimentaire des influences des variations climatiques sont ici mis en évidence.
\end{abstract}

Mots-clés : Charente, karst, spéléologie, remplissages karstiques, datations U/Th et ${ }^{14} \mathrm{C}$, sédimentologie, géochimie, analyse micro fluorescence de rayon $\mathrm{X}$, granulométrie, minéralogie des sables et des argiles, caractérisation de faciès, effet de site

\section{ABSTRACT}

MORPHOSEDIMENTARY STUDY OF A CAVE DEPOSIT AT BOIS DU CLOS CAVE (CHARENTE, FRANCE): GEOMETRIC CONSTRAINTS AND HYDROLOGICAL DYNAMICS

A section in a remarkable sequence of detrital deposits of more than $5 \mathrm{~m}$ high, filling in the cave of the Clos du Bois (La Rochefoucauld karst, Charente) allowed a morphosedimentary analysis of a discontinuous and complex sequence. Three major phases of submergence / dewatering of the cave have been identified by the combination of a grain-size analysis, Passega diagrams, a mineralogical analysis of sands and clays, and a geochemical analysis. Despite the lack of chronological milestones within the sedimentary sequence, a chronostratigraphy and time setting are proposed following the study of a stalagmite that seals the filling and is dated from marine isotopic stage 3 . The results are correlated with episodes highlighted in nearby sites. They show in our case the palaeofunctioning of an initially open karst system heavily influenced by the fluctuation of a large karst water table (the Touvre) and by nearby flows (palaeostreams and palaeovadose flows), but gradually closing down. The palaeoclimatic and palaeoenvironmental interpretation of the sediments is discussed. Notions of endokarstic trap and sedimentary gap are developed. The "site effects" on the sedimentary record of the influences of climate variability are shown.

Key-words: Charente, karst, cave deposits, U/Th and ${ }^{14} \mathrm{C}$ datings, sedimentology, geochemistry, micro X-ray fluorescence, grainsize analysis, clay and sands mineralogy, facies characterization, site effect

\section{1 - INTRODUCTION}

Si les sédiments détritiques endokarstiques sont reconnus comme étant des témoins privilégiés de l'évo- lution géomorphologique d'une région, de la karstogenèse d'un massif et de la spéléogenèse (Renault, 1967; Ek \& Quinif, 1988; Maire, 1990; Debard, 1997; Delannoy, 1997; Delannoy \& Caillault, 1998), leur

\footnotetext{
${ }^{1}$ ADES, UMR 5185 CNRS - Bordeaux 3, Maison des Suds, 12 esplanade des Antilles, F-33607 PESSAC cedex.

Courriels: gregory.dandurand@gmail.com, rmaire@ades.cnrs.fr

${ }^{2}$ CENBG, UMR 5084 CNRS, Chemin du Solarium, F-33175 GRADIGNAN, Courriels : deves@cenbg.in2p3.fr, ortega@cenbg.in2p3.fr

3 LSCE, UMR CEA - CNRS - UVSQ1572, Bâtiment 701, l'Orme les Merisiers CEA Saclay, F-91198 GIF-SUR-YVETTE cedex. Courriel: dominique.genty@1sce.ipsl.fr

${ }^{4}$ GEOTOP - UQAM - McGill, Université du Québec, Case postale 8888, Succursale A, Montréal, Québec, H3C 3P8, Canada.

Courriel: ghaleb.bassam@uqam.ca
} 
intérêt paléoenvironnemental et paléoclimatique n'atteint pas encore les résultats à très haute résolution obtenus à partir de l'analyse des enregistrements polliniques, des lamines de croissance par fluorescence $\mathrm{X}$ et des variations isotopiques du $\delta^{18} \mathrm{O}$ et du $\delta^{13} \mathrm{C}$ des spéléothèmes échantillonnés sur tous les continents (Duplessy et al., 1972; Atkinson et al., 1978; Bastin, 1990; Gascoyne, 1992; Genty \& Deflandre, 1998; Perrette, 1999; Genty et al., 2001; Genty, 2002; Genty et al., 2003; Vaks et al., 2003; Drysdale et al., 2004; McDermott, 2004; Blanc, 2005; Couchoud, 2006; Fairchild et al., 2007; Fairchild \& Treble, 2009). Un certain nombre de paramètres limite les interprétations paléoclimatiques des dépôts détritiques endokarstiques: la difficulté d'application des méthodes de datations numériques, l'influence des paramètres locaux comme la place du remplissage dans le réseau souterrain, la nature de l'encaissant calcaire, la géomorphologie du site, le volume des galeries et des salles, et des dépôts parfois stériles d'un point de vue pollinique ou paléontologique. Ainsi plusieurs auteurs restreignent l'apport climatologique et environnemental des dépôts détritiques tant en entrée de grottes que dans le karst profond (Campy, 1990; Quinif, 1990; Renault, 1990; Perroux, 2005 ; White, 2007). Néanmoins plusieurs études qui ont été menées sur des remplissages détritiques endokarstiques en France dans les Alpes, les Pyrénées, le Jura et en Lorraine (Maire, 1990; Quinif \& Maire, 1996; Lignier \& Desmet, 2002; Losson et al., 2006) et à l'étranger en Belgique (Quinif et al., 2006), en Autriche (Audra, 1994), en Suisse (Häuselmann, 2004), ou encore dans l'hémisphère sud en Australie (Forbes \& Bestland, 2007; Darrénougué et al., 2009) ont permis de corréler l'origine et la mise en place des sédiments à des phases climatiques régionales majeures. En réalité, les modalités de la sédimentation endokarstique dépendent directement des conditions hydrodynamiques qui elles-mêmes sont en grande partie influencées par les processus climatiques (Bögli, 1980). Les modalités de la sédimentation endokarstique sont donc indirectement dépendantes des variations climatiques (Quinif, 1994).

Mais les séquences sédimentaires ne sont pas systématiquement corrélées à des facteurs climatiques. Elles peuvent traduire également l'ouverture ou la fermeture du système karstique, l'influence des phénomènes locaux, comme les effondrements de voûte et de parois qui peuvent créer un effet barrage et former un lac dans une galerie, des processus de soutirage, de glissement, d'éboulisation... Ainsi, la morphologie et l'évolution de la cavité ont une influence majeure sur la mise en place des sédiments détritiques. Les notions de filtrage et de contrainte géométrique exercés par la morphologie de la cavité, en relation avec les régimes hydrologiques (types, origines et rythmes des écoulements), sont des notions fondamentales dans la compréhension du mode de mise en place des sédiments hypogés.

Maire \& Quinif (1988), Perroux (2005) et Lans et al., (2006) ont développé la notion de piège sédimentaire et d'effet de site dans l'endokarst profond. Perroux (2005, p. 341) a montré pour les grottes de Choranche (Vercors) et d'Orgnac (Bas-Vivarais), les perturbations des fonc- tions de «transfert et d'archivage des données environnementales et climatiques externes, via les écoulements et les particules sédimentaires $»$. La notion de piège sédimentaire nécessite de s'interroger sur le décryptage des signaux paléoclimatiques et paléoenvironnementaux pour évaluer et quantifier l'influence du site.

L'objectif de cette présentation est de montrer, à partir de l'exemple de la grotte du Bois du Clos, comment les caractères particuliers du site (contrainte morphologique en relation avec les régimes hydrologiques) peuvent influencer l'enregistrement sédimentaire des forçages climatiques régionaux. L'approche pluritechnique du remplissage détritique impliquant une description lithostratigraphique, une analyse granulométrique, la détermination des minéraux lourds, la morphoscopie des quartz, l'analyse minéralogique des argiles (fraction $<2 \mu \mathrm{m}$ ) associées à une analyse géochimique a fourni des résultats sur l'évolution des dynamiques hydrologiques et des contraintes géométriques du site.

\section{2 - CONTEXTE GÉOLOGIQUE ET DESCRIPTION DU REMPLISSAGE DÉTRITIQUE ÉTUDIÉ}

L'Angoumois marque le contact entre les premiers contreforts du Massif central à l'est et la bordure nordorientale du Bassin aquitain à l'ouest (fig. 1). Le karst de La Rochefoucauld s'étend sur environ $500 \mathrm{~km}^{2}$. Le bassin d'alimentation, dans lequel convergent trois rivières, la Bonnieure, la Tardoire et le Bandiat, affluents de la Charente, se développe pour l'essentiel dans les calcaires oolithiques du Jurassique moyen et supérieur (fig. 1). Une grande partie des écoulements superficiels est détournée par l'immense réseau endokarstique qui alimente la nappe phréatique. Les eaux souterraines qui drainent la structure monoclinale à pendage ouest et sud-ouest remontent au niveau de la faille de l'Echelle (Rouiller, 1987). L'exutoire est constitué par les sources de la Touvre, deuxième émergence de France par son module $\left(13 \mathrm{~m}^{3} / \mathrm{s}\right)$.

La grotte inactive du Bois du Clos se situe à $5 \mathrm{~km}$ à l'est des émergences de la Touvre. Son développement de $1200 \mathrm{~m}$, la possibilité d'avoir accès à l'aquifère, la variété des dépôts sédimentaires détritiques et carbonatés et le puissant remplissage détritique qui occupe la salle de la Boue en font une cavité unique en Charente (fig. 2).

\section{1 - GÉOMORPHOLOGIE DU SITE DE LA SALLE DE LA BOUE}

La salle de la Boue se situe à faible profondeur (- $45 \mathrm{~m}$ ) dans la partie aval du réseau du Bois du Clos au carrefour de deux galeries, les galeries des Gours et de Pâques (fig. 2). Cette salle, d'un volume important (12 $\mathrm{m}$ de diamètre et $19 \mathrm{~m}$ de hauteur) ne communique pas directement avec les lacs situés $10 \mathrm{~m}$ en aval. Mais en remontant sur une vire (galerie des Dunes) orientée dans l'axe prolongeant la galerie de Pâques, on atteint un puits étroit ( $<70 \mathrm{~cm}$ de diamètre) qui communique directement avec le Lac du Potier (ou salle des Lacs). 


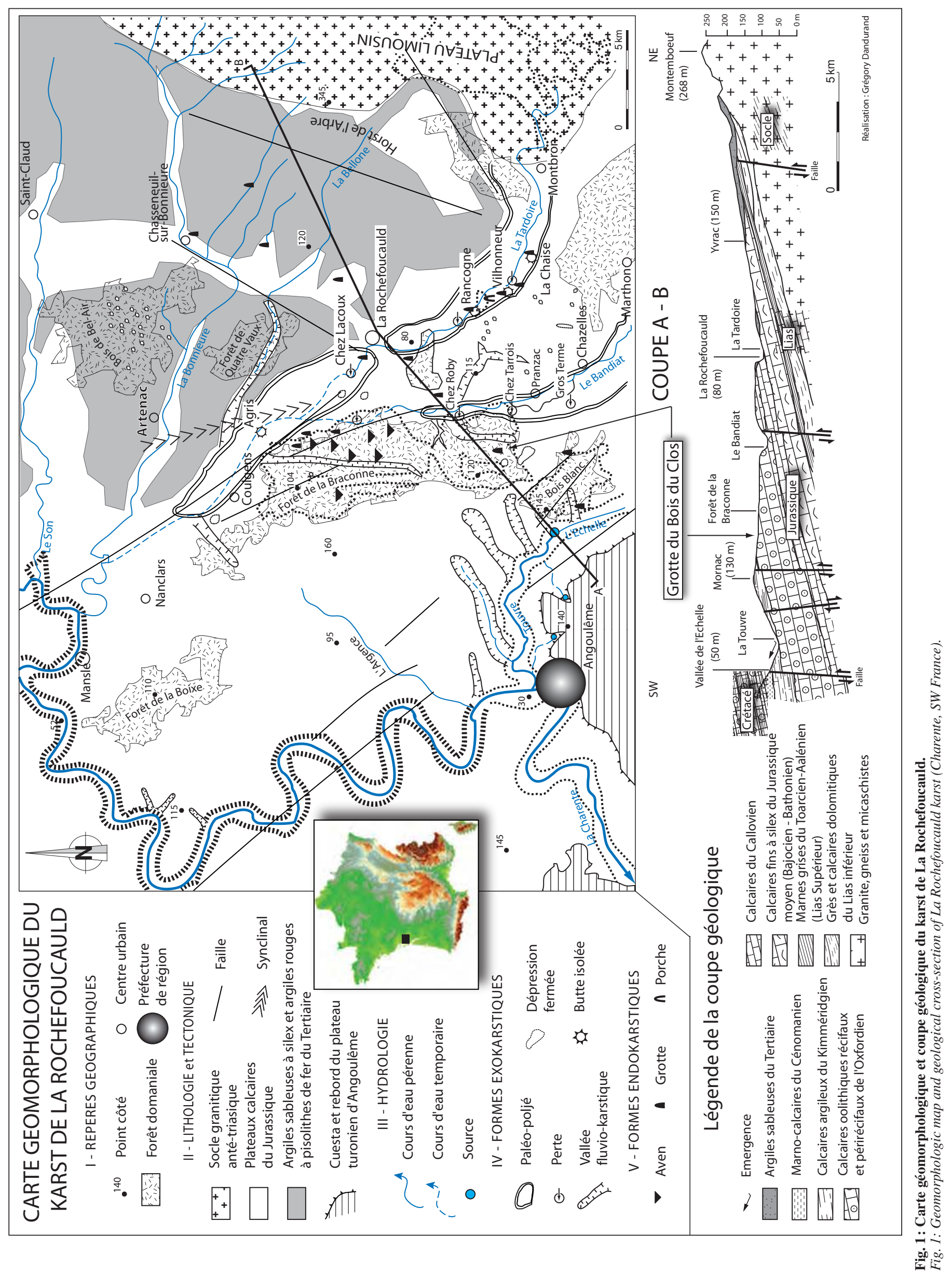




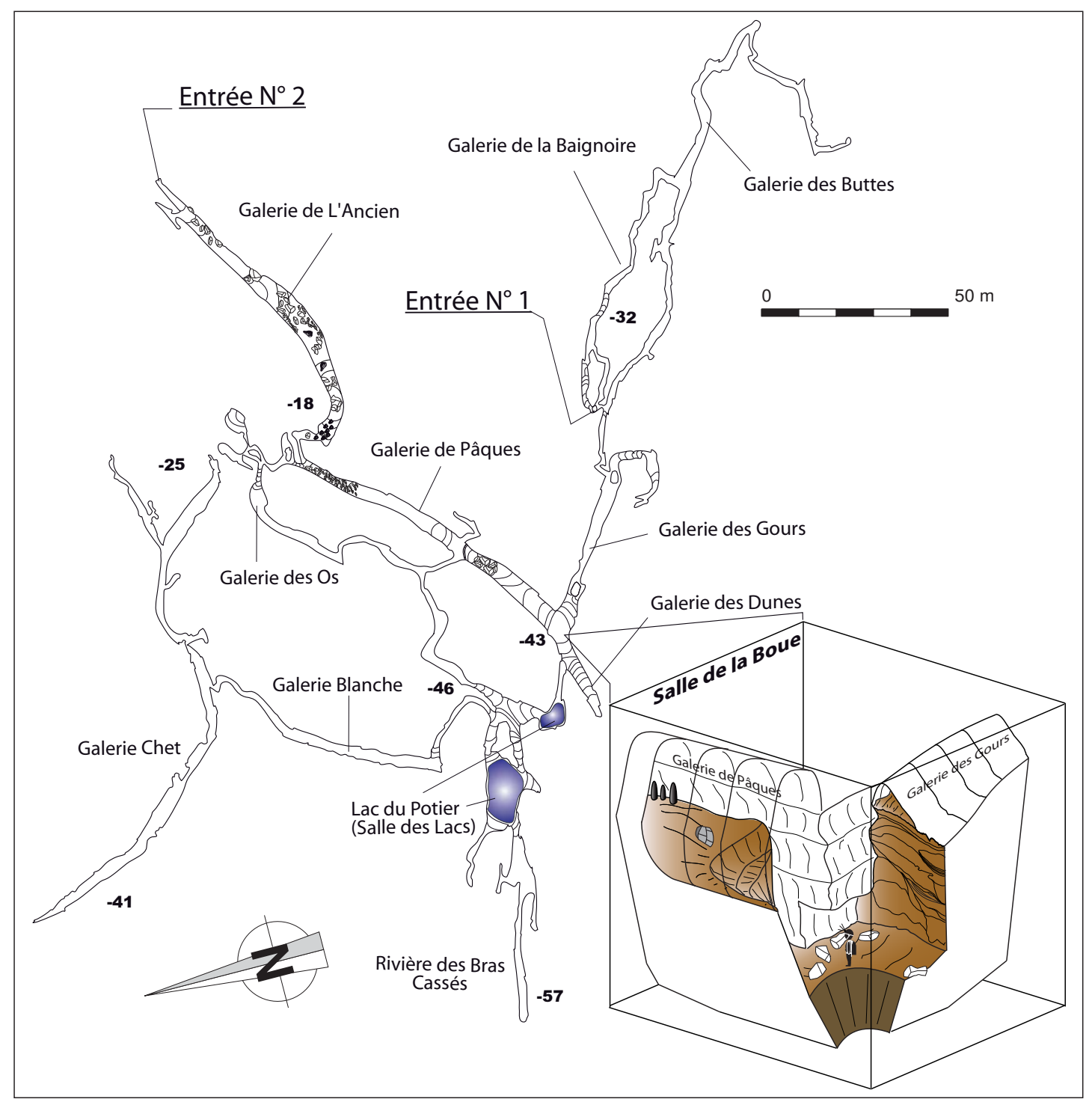

Fig. 2: Plan de la grotte du Bois du Clos (commune de Pranzac; X: 442,28; Y : 2077,74 ; Z: 110 m; relevés topographiques : J.-M. Favraud, P. Bussard, J.-M. Desvesnes, D. Doucet, C. Dupré, F. Versaveau, G. Fersing \& M. Potier) et bloc-diagramme de la salle de la Boue (observations morphométriques: G. Dandurand).

Fig. 2: Bois du Clos cave map and $3 D$ view of the Mud Room.

La profondeur reconnue du lac est de $45 \mathrm{~m}$ sans que le fond ne soit atteint. Il constitue un regard sur l'aquifère.

Les parois raides qui dominent le lac, sont tapissées d'un puissant remplissage détritique. Plusieurs dépôts similaires à ceux étudiés dans la salle de la Boue y ont été reconnus. L'ensemble inférieur (unités A, B, C et D) du remplissage de la salle de la Boue a été identifié dans la salle des Lacs, à la même altitude. L'analyse granulométrique a permis de vérifier ces similitudes de faciès. Les deux salles devaient donc fonctionner conjointement. Mais la sédimentation des deux salles n'est que partiellement commune. En effet, les unités des ensembles moyen et supérieur de la séquence de la salle de la Boue (unités D' à L) n'apparaissent pas dans la salle des Lacs.

Le site de la salle de la Boue a été favorable à l'accumulation d'une puissante séquence détritique de plus de 15 m d'épaisseur. Les 2/3 inférieurs ne sont pas accessibles en raison d'un soutirage qui a basculé et rendu inaccessible les couches détritiques inférieures. Néanmoins, le dégagement des $5 \mathrm{~m}$ supérieurs montre que la salle de la Boue a constitué un piège sédimentaire remarquable, accumulant des dépôts plus ou moins rythmés limonoargileux et constitués en partie de niveaux laminés et de chenaux sableux alimentés à la fois par un battement de la nappe phréatique du bassin de la Touvre et des écoulements proches et concentrés à partir de pertes locales de surface. Le dépôt a été ensuite scellé par un plancher stalagmitique hérissé de quelques stalagmites et finalement raviné (entonnoir de soutirage).

\section{2 - LE LEVÉ DE LA COUPE SÉDIMENTAIRE}

Le levé de la coupe sédimentaire a été réalisé sur la face orientale de la salle, dans l'axe de la galerie des Gours (fig. 3). L'ensemble de la séquence détritique n'est pas accessible: la partie inférieure reste encore invisible. La coupe mesure 5,63 $\mathrm{m}$ de haut et $4 \mathrm{~m}$ de large.

L'échantillonnage a été réalisé par le biais de $U$-channels dans le but de réaliser un profil physico-chimique à haute résolution de la séquence sédimentaire. Les $U$-channels 


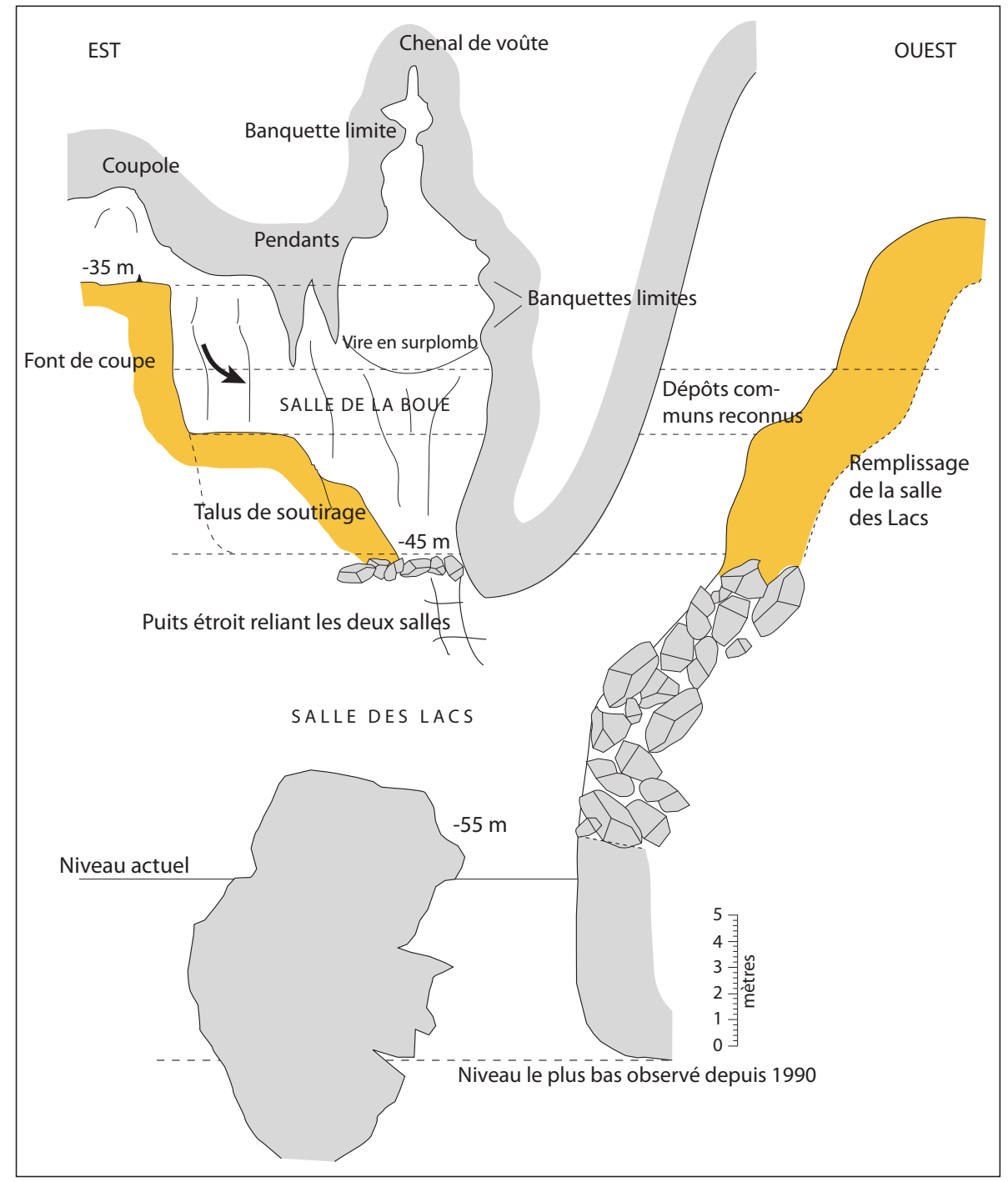

Fig. 3 : Coupe transversale des salles de la Boue et des Lacs.

Fig. 3: Cross-section of the Mud and Lakes Room.

que nous avons utilisés sont des profilés aluminium en «U» de $30 \mathrm{~cm}$ de long, $2 \mathrm{~cm}$ de profondeur et $2 \mathrm{~cm}$ de large. La technique consiste à positionner et à enfoncer les profilés perpendiculairement aux structures sédimentaires mises en évidence par la coupe. Afin d'assurer la continuité de l'échantillonnage, les profilés se chevauchent de quelques centimètres. L'extraction est réalisée à l'aide d'un couteau utilisé en biseau le long de chaque U-channel. Le surplus de sédiments est retiré à l'aide d'un fil à couper le beurre. Cette technique d'échantillonnage n'est pas fréquemment employée (Perroux, 2005; Antoine et al., 2009). Néanmoins elle présente un certain nombre d'avantages. Elle permet de conserver la structure du dépôt et de faire un prélèvement en continu de la séquence sédimentaire. Au total, 57 U-channels, répartis en trois colonnes (A, B et C) ont été extraits. Pour cette étude seule la colonne $\mathrm{B}$ a été utilisée.

\section{3 - LITHOSTRATIGRAPHIE DU REMPLISSAGE DE LA SALLE DE LA BOUE}

La séquence décrite ci-dessous a été réalisée à partir d'une coupe sédimentaire unique située dans la salle de la Boue. Le levé (fig. 4 et 5) présente trois ensembles stratigraphiques, de la base au sommet: un ensemble inférieur, un ensemble moyen et un ensemble supérieur et un plancher hérissé de stalagmitiques qui scellent le remplissage détritique.

- L'ensemble inférieur débute à la base par une poche de sables grossiers dont la partie inférieure n'a pu être dégagée. Haute de quelques dizaines de centimètres, elle est surmontée par une puissante série de trois couches métriques compactes et massives de limons argileux. Aucune lamination n'a été reconnue. La première couche correspond à une formation limono-argileuse jaune plastique et collante, séparée par une discordance nette avec l'unité stratigraphique supérieure. Celle-ci est une formation grise très compacte présentant une surface de ravinement et recoupée par un chenal remarquable. La troisième couche est une formation rouge visiblement érodée et aussi entaillée par le chenal sur la droite.

- L'ensemble moyen est l'ensemble principal de la coupe. Il peut se subdiviser en deux sous-ensembles. Il débute à la base par des limons fins rouges qui occupent le fond du chenal évoqué ci-dessous. Par-dessus vient une puissante formation de sables grossiers jaunes lités (asymétrie), présentant des déformations (micro-failles, 


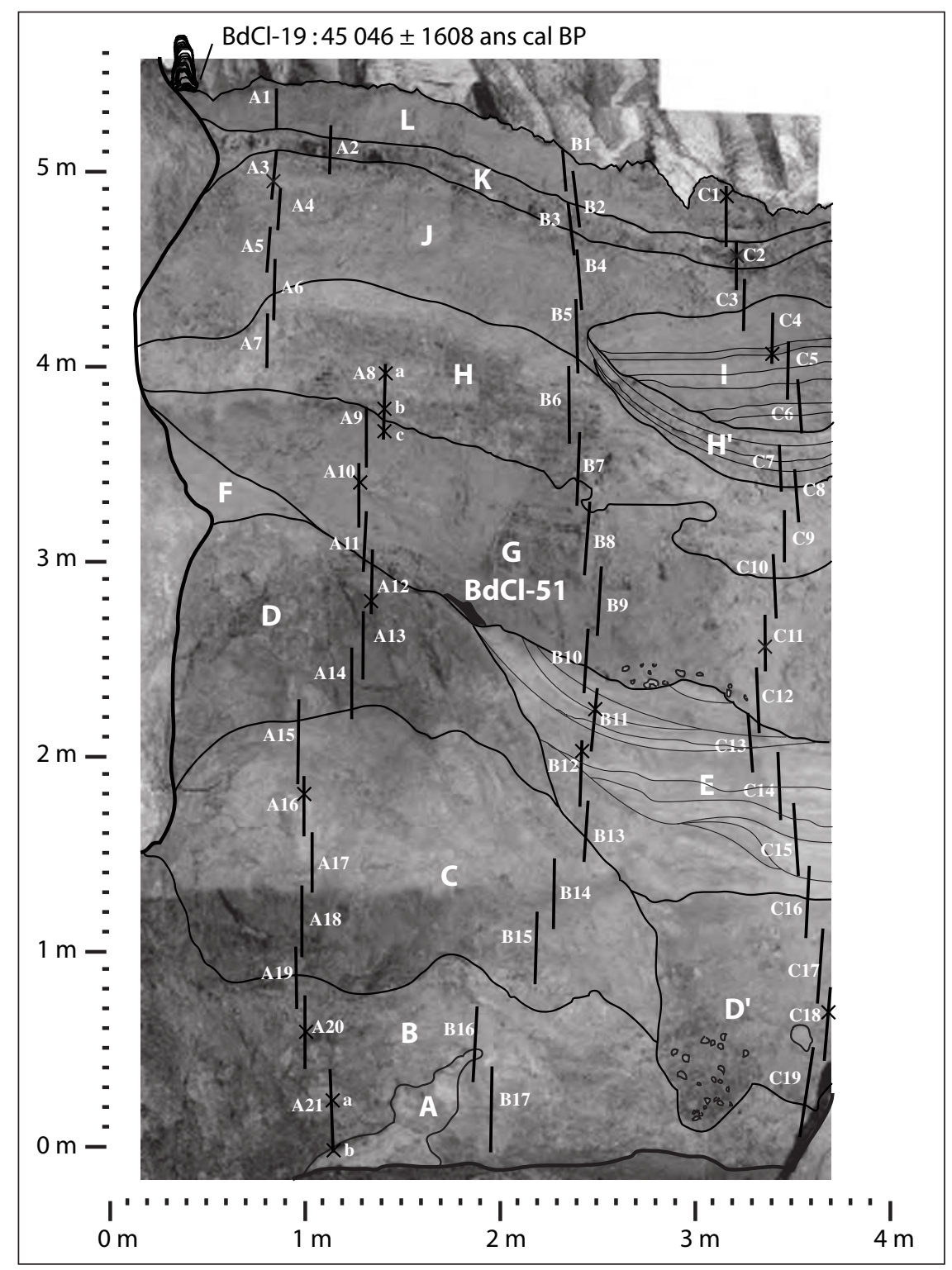

Fig. 4 : Coupe dans le remplissage détritique de la salle de la Boue dans l'axe de la galerie des Gours.

14 unités stratigraphiques ont été mises en évidence. 2 spéléothèmes ont été prélevés au centre et au sommet de la séquence. Celle-ci présente de nombreuses discontinuités et surfaces de ravinement.

Fig. 4: Section of the sedimentary sequence infilling the Mud Room. 14 stratigraphic units were identified. 2 speleothems were collected in the center and top of the sequence, which shows many discontinuities and surfaces of erosion.

dépôts lenticulaires érodés et biseautés). Une stalagmite (BdCl-51) basculée et corrodée ainsi que des limons fins résiduels érodés ont été reconnus au-dessus de la formation sableuse. La transition avec le second sousensemble se fait par une discordance très nette. Les dépôts sus-jacents correspondent à deux séries de varves très finement laminées. La première se caractérise par une couleur rouge tandis que la seconde est jaune. Inclinées vers la droite de la coupe, elles présentent chacune une surface ravinée.

- L'ensemble supérieur débute par un chenal de sables bruns moins épais que le précédent et légèrement décalé sur la droite de la coupe. Il recoupe de façon moins prononcée l'unité sous-jacente. Il se caractérise par des dépôts grossiers lités disposés en couches centimétriques séparés par des lits plus limoneux dont l'un de couleur rouge se distingue nettement. Il est surmonté par un puissant dépôt de galets mous limoneux qui repose en discordance sur les dépôts sableux et sur les varves jaunes de l'ensemble moyen. La séquence se termine par une bande argilo-limoneuse jaune brun très compacte, de $22 \mathrm{~cm}$ d'épaisseur, incurvée sur la droite de la coupe. Elle est recouverte par un dépôt de limons argileux marron, contenant des billes de quartz et de calcaires. Le remplissage détritique est scellé par un plancher hérissé de stalagmites. Il est à noter que le remplissage s'est tassé de quelques centimètres, attesté par le vide entre le plancher stalagmitique et les dépôts sommitaux.

\section{4 - LES CONCRÉTIONS STALAGMITIQUES}

Les stalagmites qui scellent le remplissage sont actives (fig. 3). Dans cet article nous nous intéresserons uniquement à l'une d'entre elle. Haute de $25 \mathrm{~cm}$, la stalagmite BdCl-19 a été prélevée dans le réseau inférieur de la galerie de Pâques, à moins deux mètres du soutirage de 


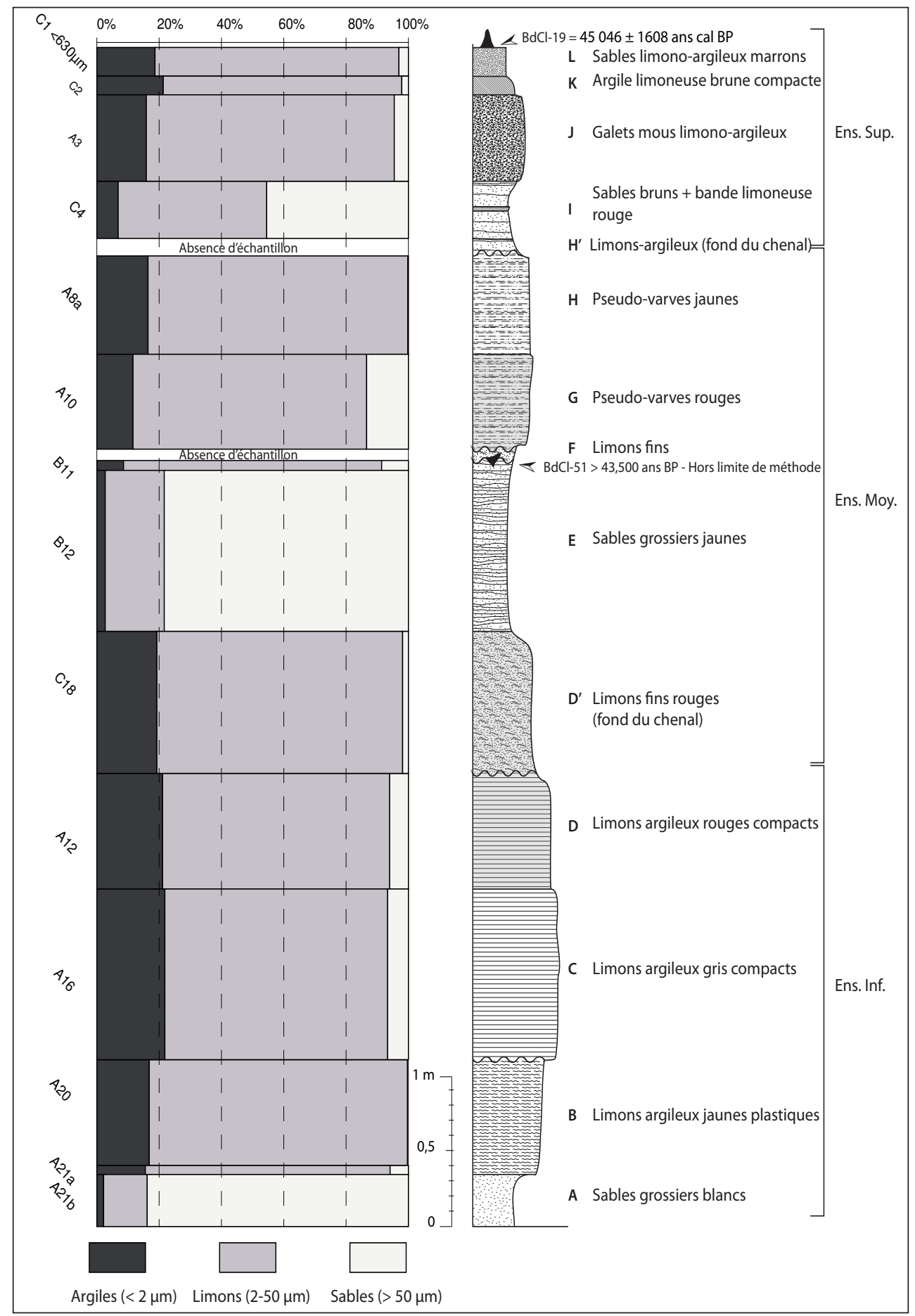

Fig. 5 : Levé de synthèse et granulométrie de la séquence sédimentaire. Fig. 5: Synthetic and granulometry of the sedimentary sequence.

la salle de la Boue. La stalagmite s'est développée postérieurement au remplissage de la salle de la Boue. Elle constitue donc un marqueur chronologique précieux.

L'autre concrétion située à l'intérieur du remplissage a également été prélevée afin de caler chronologiquement les ensembles moyen et supérieur.

\section{5 - GÉOMÉTRIE DU REMPLISSAGE ET MORPHO- LOGIE DE LA SALLE DE LA BOUE}

Leétude de la géométrie des dépôts met en évidence au moins quatre discordances majeures. Hormis la poche sableuse à la base, l'ensemble inférieur se caractérise par des couches sédimentaires massives disposées horizontalement. Une surface d'érosion irrégulière a été reconnue entre l'unité B et l'unité C. La limite supérieure de l'unité $\mathrm{B}$ devait s'arrêter à hauteur de la paroi sur la gauche de la coupe. Elle a été érodée sur $70 \mathrm{~cm}$. Au-dessus les couches ne présentent pas de lamination. Une autre surface d'érosion bien visible recoupe les unités $\mathrm{F}, \mathrm{D}$ et $\mathrm{E}$. Inclinée à $33^{\circ}$ vers le sud de la salle, elle va influencer la géométrie du remplissage. Les couches des ensembles moyen et supérieur ont un pendage sud de $20^{\circ}$. Ces variations de pendage révèlent un encaissement et un soutirage progres- 
sifs de la séquence vers le sud. Le décalage progressif des niveaux sableux étagés vers le sud corrobore cette idée.

Plusieurs indices nous permettent de penser que la séquence sédimentaire s'est mise en place dans une salle aux caractéristiques morphologiques semblables à l'actuelle. Les banquettes latérales repérées au niveau des couches F et K n'ont pas été reconnues en symétrie sur la paroi opposée. De plus, le renfoncement en bas à gauche de la coupe et le pendant de voûte corrodé en haut à droite sont significatifs. Le toit de l'encaissant est en contact direct avec l'unité B. Sa surface plane au même niveau que le toit de l'unité $\mathrm{B}$, indique qu'il préexistait à la sédimentation de l'unité B. Malgré la présence de morphologies telles que des chenaux de voûte, des banquettes latérales, des marmites de plafond, des pendants de voûte, l'hypothèse d'une genèse noyée de type paragénétique, c'està-dire formée par corrosion remontante au-dessus d'un remplissage de décantation progradant (Renault, 1967) est ici exclue. Le pendant de voûte corrodé au sommet du remplissage a contraint la sédimentation de l'unité L sans l'empêcher. La spéléogenèse doit donc être antérieure au remplissage détritique (Dandurand \& Maire, 2011).

\section{3 - MÉTHODES ET RÉSULTATS}

L'analyse sédimentologique a été traitée de trois manières, d'un point de vue granulométrique, minéra- logique et géochimique. L'utilisation d'un triangle de texture et les analyses granulométriques ont permis de caractériser la nature des dépôts détritiques; les résultats replacés sur un diagramme de Passega (fig. 6) et croisés avec des analyses minéralogiques des sables et des argiles et de la morphoscopie des grains de quartz ont permis de mettre en évidence l'évolution des dynamiques hydro-sédimentaires dans la cavité et l'origine des sédiments. Enfin, l'analyse géochimique, corrélée à la granulométrie, a permis de confirmer la discrimination des différentes unités stratigraphiques. Finalement, c'est la comparaison des résultats avec ceux obtenus dans des sites karstiques proches qui permettra de discriminer l'influence des paramètres locaux de celle des paramètres régionaux.

\section{1 - LES ANALYSES GRANULOMÉTRIQUES}

L'analyse granulométrique repose sur l'interprétation à partir d'un triangle des textures et sur une étude statistique de la fréquence des tailles de grains dans un sédiment (histogrammes de fréquence et courbes semi-logarithmiques cumulatives). Pour les éléments les plus fins $(<900 \mu \mathrm{m})$, un microgranulomètre laser (modèle MALVERN@ Master Size S.) a été utilisé (resp. G. Chabaud, lab. EPOC Bordeaux 1). Plusieurs auteurs ont appliqué ces méthodes statistiques et graphiques à l'étude des sédiments du karst profond (Jeannin et al., 1990; Maire, 1990; Quinif, 1990;

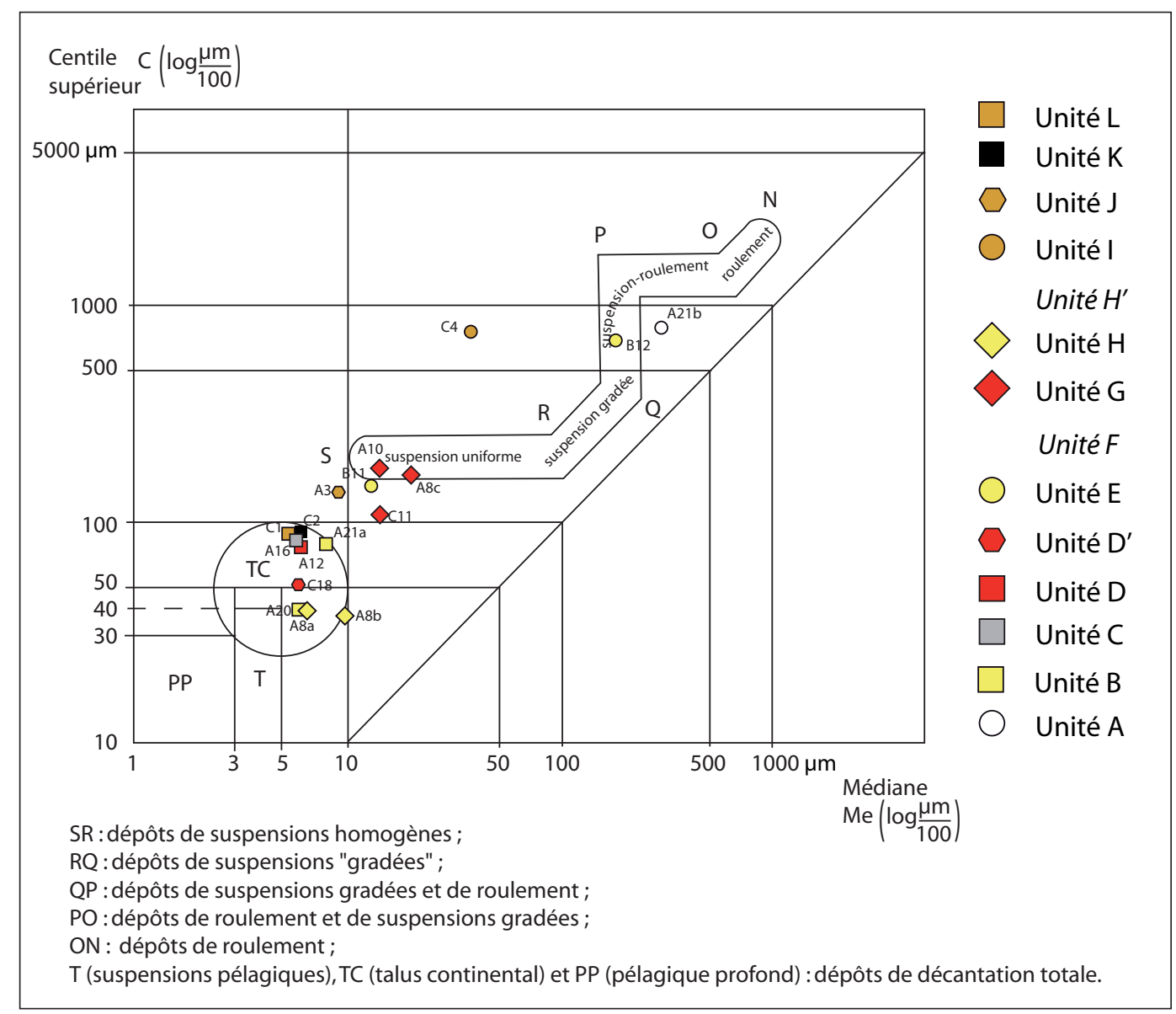

Fig. 6: Unités stratigraphiques replacées dans un diagramme de Passega.

Fig. 6: Stratigraphic units represented in a Passega diagram. 
Renault, 1990; Audra, 1994; Ferrier, 1994; Pomel \& Maire, 1995, 1997; Héraud-Piña, 1996; Debard, 1997; Delannoy \& Caillault, 1998; Le Fillâtre, 2001; Lignier \& Desmet, 2002; Losson, 2003; Perroux, 2005). Dans cet article, les seuls paramètres granulométriques (tab. 1) utilisés sont le mode, la médiane (C50) et le centile supérieur (C99). Seul l'indice granulométrique Skewness (Sk) est ici présenté. Il s'agit d'un coefficient de dissymétrie. Il est exprimé en échelle $\Phi$. Sk = Q75.Q25/Me ${ }^{2}$. Si Sk est égal à 1 , on a alors une courbe symétrique. Lorsque Sk est $<1$, la dissymétrie est en faveur des éléments grossiers; inversement quand Sk est $>1$.

L'utilisation d'un diagramme de Passega (1964) est généralement appliquée à des sédiments marins et fluviatiles. Le diagramme, sur une échelle bilogarithmique, exprime les valeurs du centile supérieur en fonction de la médiane. Il permet de distinguer plusieurs secteurs ou segments correspondant à des types particuliers de sédiments et donc à des modes différents de transports. Les dépôts de type «suspension uniforme» correspondent à des matières en suspension sur toute la colonne d'eau. Leur sédimentation se produit par décantation des eaux turbides, lorsque la vitesse au fond du cours d'eau est nulle ou trop faible pour produire un classement. Les dépôts de type «suspension gradée» renvoient à des matériaux classés, transportés par des courants de fond qui donnent une sédimentation typique dans les chenaux fluviatiles. La taille des sédiments est décroissante vers le haut. Les dépôts de type «roulement» caractérisent, quant à eux, des sédiments déposés dans des conditions où les courants de fond sont plus rapides. Seuls les éléments plus grossiers sont roulés et les suspensions ne peuvent plus sédimenter. Enfin, les dépôts de type «suspension pélagique», «talus continental» et «pélagique profond»correspondent aux particules les plus fines (médiane $<10 \mu \mathrm{m}$ et centile supérieure $<100 \mu \mathrm{m}$ ), qui sous l'influence des ions floculants peuvent être transportés sur une longue distance et finissent par se déposer dans des conditions de décantation totale. Cette méthode, employée depuis longtemps en karstologie (Quinif et al., 1979; Maire \& Quinif, 1988; Audra, 1994; Perroux, 2005), a permis dans notre cas de préciser les régimes hydrodynamiques qui sont à l'origine des dépôts dans la salle de la Boue.

\section{2 - LA MINÉRALOGIE DES SABLES ET DES ARGILES}

La minéralogie des sables repose sur l'identification, l'observation et la quantification des minéraux lourds contenus dans un échantillon. La reconnaissance d'un cortège minéralogique précis permet de connaître l'ori-

\begin{tabular}{|c|c|c|c|c|c|c|c|c|c|}
\hline \multirow{2}{*}{$\begin{array}{l}\text { Unité } \\
\text { stratigra } \\
\text { phique }\end{array}$} & \multirow{2}{*}{$\begin{array}{l}N^{\circ} \\
\text { éch. }\end{array}$} & \multirow{2}{*}{$\begin{array}{l}\text { Nature du } \\
\text { sédiment }\end{array}$} & \multirow{2}{*}{$\begin{array}{c}\text { Indice } \\
\text { granulométrique } \\
\text { Skewness }(S k, \\
\text { exprimé en } \Phi)\end{array}$} & \multicolumn{4}{|c|}{$\begin{array}{l}\text { Paramètres granulométriques } \\
\text { (données brutes en } \mu \mathrm{m} \text { ) }\end{array}$} & \multirow{2}{*}{$\begin{array}{l}\text { Diagramme de } \\
\text { Passega }\end{array}$} & \multirow{2}{*}{ Dynamique hydrologique } \\
\hline & & & & Mode & Dév. St. & $\begin{array}{c}\mathrm{Me} \\
\text { (C50) }\end{array}$ & Cs (C99) & & \\
\hline L & C1 & Limons argileux & -0.10 & 4.9 & 1.7 & 5.4 & 90 & $\begin{array}{l}\text { "suspension } \\
\text { pélagique" }\end{array}$ & décantation totale \\
\hline $\mathrm{K}$ & C2 & Limons argileux & -0.10 & 4.2 & 1.7 & 6 & 90 & $\begin{array}{l}\text { "suspension } \\
\text { pélagique" }\end{array}$ & décantation totale \\
\hline$J$ & A3 & Limons argileux & 0.09 & 12.2 & 1.8 & 9 & 140 & $\begin{array}{l}\text { proche } \\
\text { suspension } \\
\text { uniforme }\end{array}$ & décantation d'eau turbide \\
\hline 1 & C4 & Limons sableux & 0.25 & 477.0 & 2.9 & 38 & 780 & $\begin{array}{l}\text { Proche } \\
\text { suspension- } \\
\text { roulement }\end{array}$ & $\begin{array}{l}\text { présence d'éléments fins qui } \\
\text { diminuent la médiane -> dépôt } \\
\text { fluviatile }\end{array}$ \\
\hline $\mathrm{H}$ & A8a & Limons argileux & 0.32 & 12.2 & 1.5 & 6.3 & 40 & $\begin{array}{l}\text { "suspension } \\
\text { pélagique" }\end{array}$ & décantation totale \\
\hline $\mathrm{H}$ & A8b & Limons très fins & 0.85 & 14.2 & 1.4 & 9.9 & 37 & $\begin{array}{l}\text { "suspension } \\
\text { pélagique" }\end{array}$ & décantation totale \\
\hline $\mathrm{G}$ & $\mathrm{A} 8 \mathrm{c}$ & Limons fins & 0.59 & 35.6 & 1.8 & 20 & 165 & $\begin{array}{l}\text { suspension } \\
\text { uniforme }\end{array}$ & décantation d'eau turbide \\
\hline $\mathrm{G}$ & $\mathrm{C} 11$ & Limons argileux & 0.51 & 26.2 & 1.7 & 14 & 110 & $\begin{array}{l}\text { proche } \\
\text { suspension } \\
\text { uniforme }\end{array}$ & décantation d'eau turbide \\
\hline $\mathrm{G}$ & A10 & Limons fins & 0.34 & 26.2 & 1.9 & 14 & 180 & $\begin{array}{l}\text { suspension } \\
\text { uniforme }\end{array}$ & décantation d'eau turbide \\
\hline E & B11 & Limons très fins & 0.40 & 19.3 & 1.7 & 13 & 150 & $\begin{array}{l}\text { proche } \\
\text { suspension } \\
\text { uniforme }\end{array}$ & décantation d'eau turbide \\
\hline E & $\mathrm{B} 12$ & Sables limoneux & 1.38 & 259.0 & 2.2 & 180 & 720 & $\begin{array}{l}\text { suspension } \\
\text { gradée }\end{array}$ & $\begin{array}{l}\text { dépôt fluviatile ; courant } \\
\text { moyen à fort }\end{array}$ \\
\hline$D^{\prime}$ & $\mathrm{C} 18$ & Limons argileux & 0.10 & 3.6 & 1.7 & 6 & 52 & $\begin{array}{l}\text { "suspension } \\
\text { pélagique" }\end{array}$ & décantation totale \\
\hline $\mathrm{D}$ & A12 & Limons argileux & -0.03 & 3.1 & 1.9 & 6 & 80 & $\begin{array}{l}\text { "suspension } \\
\text { pélagique" }\end{array}$ & décantation totale \\
\hline C & A16 & Limons argileux & -0.08 & 3.1 & 1.9 & 5.9 & 84 & $\begin{array}{l}\text { "suspension } \\
\text { pélagique" }\end{array}$ & décantation totale \\
\hline B & A20 & Limons argileux & 0.38 & 9.0 & 1.5 & 6 & 40 & $\begin{array}{l}\text { "suspension } \\
\text { pélagique" }\end{array}$ & décantation totale \\
\hline B & A21a & Limons argileux & 0.26 & 22.5 & 1.8 & 8 & 80 & $\begin{array}{l}\text { "suspension } \\
\text { pélagique" }\end{array}$ & décantation totale \\
\hline A & $A 21 b$ & Sables limoneux & 1.84 & 409.4 & 2.2 & 300 & 810 & $\begin{array}{l}\text { proche } \\
\text { suspension } \\
\text { roulement }\end{array}$ & $\begin{array}{l}\text { dépôt fluviatile ; courant } \\
\text { moyen à fort }\end{array}$ \\
\hline
\end{tabular}

Tab. 1 : Paramètres et indices granulométriques.

Tab. 1: Parameters and particle size index. 
gine et la provenance des sédiments. Elle est complétée par la morphoscopie des quartz, reposant sur l'étude de la forme et de l'aspect de surface des grains de quartz permet de distinguer trois types: les grains non usés (NU), anguleux, transparents ou colorés ils ont subi un faible transport; les émoussés-luisants (EL), sub-anguleux, polis et brillants, ils traduisent un transport fluviatile; les ronds-mats (RM) ovoïdes ou arrondis, à surface picotée et mate, ils correspondent à un transport éolien (Cailleux \& Tricart, 1959).

Deux techniques de préparation ont été mises en œuvre. Dans la première, l'échantillon est dilué dans de l'eau. Les grains sont séparés par décantation. Puis ils sont nettoyés à l'acide chlorhydrique (35\%), puis séchés et enfin triés par taille au tamis. La partie la plus grossière est subdivisée en deux types de taille $(\geq 160 \mu \mathrm{m}$ et $100-160 \mu \mathrm{m}$ ). Les sablons (partie la plus fine) sont compris entre $0,63 \mu \mathrm{m}$ et $100 \mu \mathrm{m}$. Les grains, fixés par du baume du Canada, sont ensuite placés sous lame mince. La seconde technique consiste à imprégner les sédiments par une résine polyester (types GBS1 Brot Technologies ${ }^{\circledR}$ et SINTOFER ${ }^{\circledR}$, Gaine, 1973; Crovisier, 1979), les échantillons sont préparés pour lame mince par J.-C. Pellegrin (lab. LGPA) et M. Cremer (lab. EPOC). L'observation des minéraux en grains a été réalisée à l'aide d'une loupe binoculaire et d'un microscope polarisant, modèle Zeiss Axioplan 2, au laboratoire ADES de la Maison des Suds à Pessac.

L'analyse minéralogique des argiles par diffractométrie des rayons $\mathrm{X}$ a été effectuée au laboratoire HydrASA (UMR CNRS 6269 / Université de Poitiers) par l'intermédiaire de A. El Albani. Quatre échantillons (B17, A16, A9 et C2) associés aux unités stratigraphiques B, $\mathrm{C}$, D et $\mathrm{K}$ ont pu être analysés. Les diffractogrammes obtenus à partir de la fraction $<2 \mu \mathrm{m}$ sur lames orientées (raies $00 \ell$ ) naturelles séchées à l'air ont été réalisés à partir d'un diffractomètre Siemens avec une radiation $\mathrm{CuK} \alpha$. Les conditions expérimentales employées sont un balayage de 2,5 à $35^{\circ} 2 \theta$ avec un pas de temps de $0,02^{\circ}$ en $2 \theta$ et un temps de comptage de 5 secondes. Lidentification et l'estimation quantitative des minéraux argileux (Holtzapffel, 1985; Caner, 2011) ont été réalisées à partir de la position et de l'intensité (ou distance réticulaire) des pics (d001).

L'analyse minéralogique des argiles a mis en évidence dans les quatre échantillons la présence de quatre types de minéraux : chlorite, smectite, illite et kaolinite (fig. 7). En outre, des minéraux non argileux ont été identifiés. Des grains de quartz ont été détectés dans les échantillons $\mathrm{C} 2$, A9 et A16, de goethite dans les échantillons A16 et B17, de feldspaths potassiques dans B17 et $\mathrm{C} 2$ et de calcite dans l'échantillon C2. Minéraux argileux et non argileux sont des marqueurs précieux des processus pédogénétiques, en lien avec les conditions paléoenvironnementales et paléoclimatiques, qui ont opéré depuis l'altération et la désagrégation de la roche dont ils sont issus (Wilson, 1999; Velde, 2008).

Le rapport que l'on peut établir entre les minéraux est un indicateur de l'évolution paléoclimatique. Le rapport kaolinites/smectites est significatif (El Albani et al.,
2010). Lorsque les kaolinites sont associées à part égale avec des smectites dans un remplissage, cela révèle des conditions relativement chaudes et sèches. En revanche, si le rapport diminue (enrichissement en smectites), cela indique une détérioration des conditions climatiques (refroidissement). En dernier lieu, une augmentation du rapport (enrichissement en kaolinites) est le signe d'une amélioration climatique (réchauffement). Ainsi, la détermination des minéraux argileux n'a de sens que si l'on prend en considération l'ensemble du cortège minéralogique et les rapports entre les minéraux. Il est aussi intéressant de relever que l'omniprésence des illites et des chlorites, toutes deux très sensibles à l'altération chimique, dans les quatre échantillons de la séquence sédimentaire indique qu'elle n'a que très peu évolué depuis son piégeage dans le karst profond.

La quantification des minéraux dans un échantillon de sédiment, malgré des progrès remarquables depuis quelques années, reste encore complexe (Caner, 2011). On considère d'ordinaire que l'intensité relative d'un minéral sur un diffractogramme ne mesure pas directement la proportion relative des différents minéraux et qu'elle varie en fonction de la composition minéralogique de l'échantillon. Lorsque le nombre de feuillets empilés de façon cohérente est élevé, la fonction d'interférence induit des pics intenses et étroits (Caner, 2011). A l'inverse, lorsque le nombre de feuillets est faible, les pics sont moins intenses et plus larges. Dans notre cas, compte tenu du fait que l'assemblage minéralogique est similaire pour les quatre unités stratigraphiques échantillonnées, on peut considérer les variations des pics d'intensité d'un échantillon à l'autre, pour un même minéral, comme étant significatives.

\section{3 - LA GÉOCHIMIE}

L'utilisation d'une microsonde XRF, appliquée aux sédiments détritiques marins et lacustres de divers contextes morpho-climatiques, a montré depuis une décennie des résultats très intéressants (Peterson et al., 2000; Böning et al., 2007; Katsuta et al., 2007; Cuven, 2009; Cuven et al., 2010; Kylander et al., 2011). En revanche, cette méthode est encore rarement appliquée à des remplissages détritiques endokarstiques. Peu d'études en karstologie s'appuient sur ce genre d'analyses (Darrénougué et al., 2009). Le choix de cette méthode dans notre étude repose sur plusieurs critères: c'est une technique innovante et encore peu employée, qui pourtant a fait des progrès remarquables ces dix dernières années (Koshikawa et al., 2003; Kido et al., 2006) tant dans la rapidité de la mise en œuvre, les étapes de préparation des échantillons et la simplicité de l'acquisition des données. Pourtant, n'ayant que peu de références dans le domaine de l'analyse géochimique en milieu endokarstique, un certain nombre de difficultés ont été rencontrées dans l'interprétation des résultats. La comparaison avec les analyses en milieu marin et lacustre a permis d'affiner la méthode. En revanche, seuls les travaux de Darrénougué et al. (2009) nous ont permis de prendre en compte dans notre interprétation les spécificités du milieu souterrain. En 


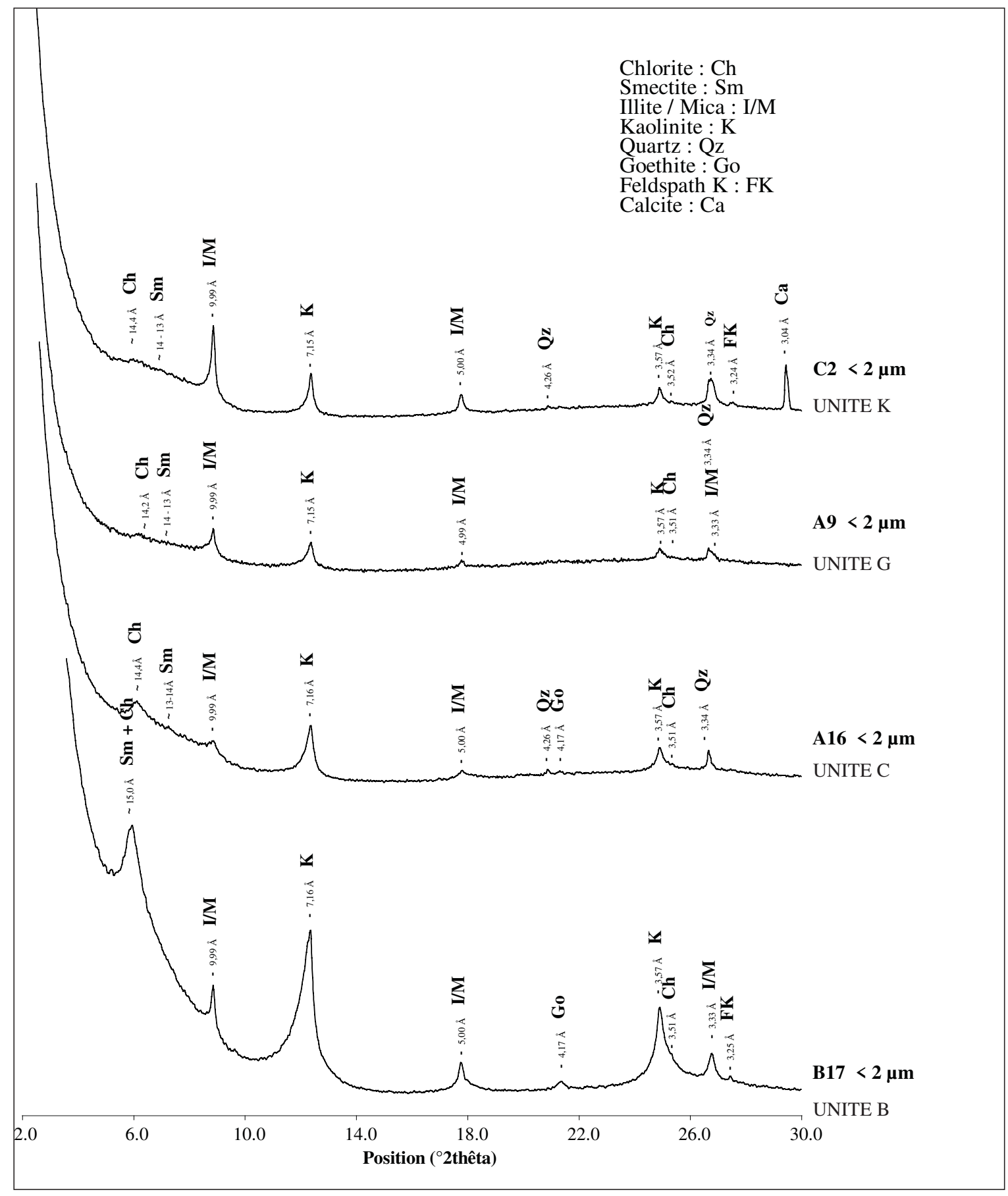

Fig. 7 : Diffractogrammes des unités B, C, G et $\mathbf{K}$ de la séquence sédimentaire du Bois du Clos.

Fig. 7: Diffractograms of units $B, C, G$ and $K$ from the sedimentary sequence of Clos du Bois.

utilisant cette technique, en corrélation avec une analyse morphoscopique et exoscopique des grains de quartz et une analyse granulométrique, Darrénougué et al. (2009) ont réussi à caractériser l'origine d'un dépôt éolien, daté entre 40 et 14 ka cal. BP, déposé dans la grotte Blanche, en Australie du Sud. Les signatures géochimiques, granulométriques et morphoscopiques des grains de quartz ont permis de mettre en évidence deux sources d'approvisionnement du stock sédimentaire, l'un en provenance de la région côtière pendant la période humide du stade isotopique marin (SIM) 3, l'autre en provenance de l'intérieur des terres pendant les phases froides et sèches du SIM 2 (Dernier Maximum Glaciaire). La rareté des études géochimiques en domaine endokarstique a donc limité les points de comparaison. C'est bien là une limite de méthode indéniable. En revanche, plusieurs auteurs ont bien caractérisé la signature géochimique de certains types de dépôts lacustres. Par exemple, Kylander et al. (2011) ont montré que la corrélation des pics de Ca et de Sr est un bon indicateur du lessivage des carbonates dans les sols du bassin-versant, avec la précipitation en milieu alcalin des carbonates en solution à partir de $\mathrm{SrCO}_{3}$. Cuven et al. (2010), Kylander et al. (2011) et Croudace et al. (2006) ont mis en évidence que les éléments Rb, K et Ti sont de bons traceurs des sédimentations de types détritiques à particules très fines, ou encore que $\mathrm{Mn}$ et $\mathrm{Fe}$ en corrélation suggèrent des processus d'oxydo-réduction. 
L'analyse géochimique a pour but de confirmer et de préciser les informations tirées de l'analyse granulométrique et de l'analyse minéralogie des sables et des argiles. En vue de réaliser un profil géochimique de la séquence stratigraphique (fig. 4), une analyse a été réalisée sur la colonne centrale de $U$-channels, la série B. Celle-ci comporte 17 U-channels sur 4,85 m de hauteur. Chaque échantillon est analysé par la microsonde XRF XGT-7000 (Horiba), au Centre d'Étude Nucléaire de Bordeaux-Gradignan.

La sonde micro-XRF (fig. 8 et 9) permet la discrimination et la quantification en continu d'éléments chimiques (Al, Si, K, Ca, Ti, Mn, Fe, Rb, Zr, Y et Zr), qui constituent des traceurs généralement utilisés dans les reconstitutions paléoenvironnementales (Peterson et al., 2000; Koshikawa et al., 2003; Kido et al., 2006; Böning et al., 2007; Katsuta et al., 2007; Cuven, 2009; Darrénougué et al., 2009; Cuven et al., 2010; Kylander et al., 2011). Le balayage de la surface des $U$-channels est réalisé par intervalles de $5 \mathrm{~mm}$ et avec un pas de temps de $300 \mathrm{~s}$. Le voltage du tube des rayons $\mathrm{X}$ est réglé à $50 \mathrm{kV}$ avec un courant de $1 \mathrm{~mA}$. Afin d'éviter des problèmes de condensation sur la lentille du détecteur il a été décidé de faire les analyses sans film plastique et de sécher au préalable les échantillons dans une étuve à $80^{\circ} \mathrm{C}$ pendant 24 heures. L'inconvénient de cette technique est la dessiccation du sédiment qui provoque une perte de volume estimée à $5 \%$. Cette réduction de volume crée parfois des fentes
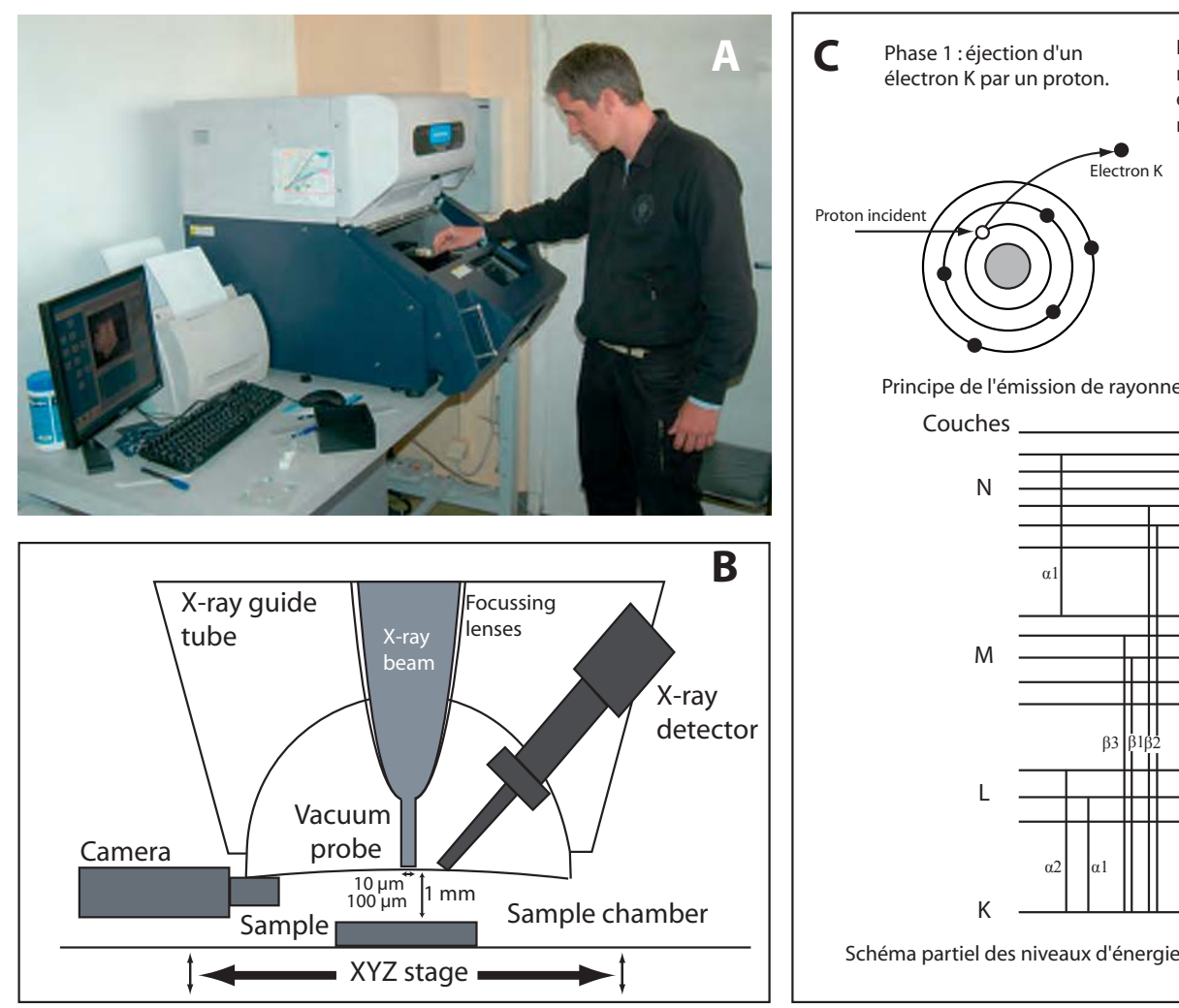

Phase 2 : Réajustement de l'arrangement électronique par transition électronique et émission d'un rayonnement X.

Principe de l'émission de rayonnement $X$ sous faisceau de protons.

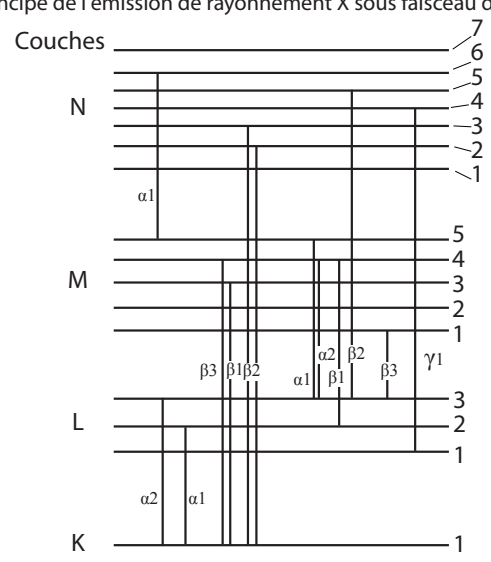

Schéma partiel des niveaux d'énergie d'un atome montrant les raies $\mathrm{K}$ et $\mathrm{L}$.

Fig. 8: Schéma et principe de la microsonde XRF Horiba XGT-7000.

(A) Photo de la microsonde XRF Horiba XGT-7000 au CENBG (Cliché R. Maire, 23/03/2009). (B) Schéma simplifié et sans échelle de la chambre de détection de la microsonde XRF (inspiré de Böning et al., 2007). (C) Principe d'excitation / désexcitation d'un électron et du rayonnement X (BellotGurlet, 1998).

Fig. 8: Schematic and principle of XRF microprobe Horiba XGT-7000. (A) Photograph of the XRF microprobe Horiba XGT-7000 at CENBG (Photo R. Maire, 23/03/2009). (B) Simplified drawing and without scale of the detection chamber of the XRF microprobe (inspired from Böning et al., 2007). (C) Principle of excitation / de-excitation of an electron and X-ray (Bellot-Gurlet, 1998). 


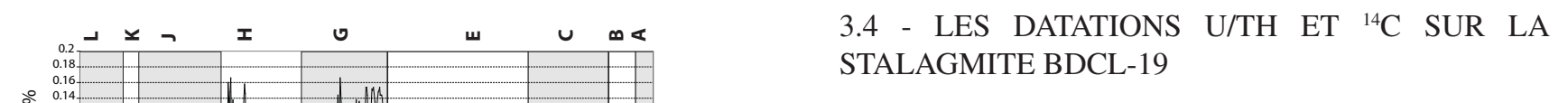

Les spéléothèmes peuvent être datés selon deux méthodes (Genty et al., 1999), la méthode du radiocarbone $\left({ }^{14} \mathrm{C}\right)$ et celle de l'uranium-thorium $(\mathrm{U} / \mathrm{Th})$ sur la calcite. La première est assez peu utilisée pour plusieurs raisons : 1) elle nécessite une correction de carbone mort (proportion de carbone issue de la dissolution de la roche encaissante et de l'activité bio-pédologique de surface) ; 2) la limite de la méthode est atteinte pour des objets vieux de plus de $40 \mathrm{ka}$ environ. La seconde méthode est la plus utilisée. Elle consiste à doser dans la calcite les isotopes de l'uranium $\left.{ }^{238} \mathrm{U},{ }^{234} \mathrm{U}\right)$ et du thorium $\left({ }^{230} \mathrm{Th}\right.$, ${ }^{232} \mathrm{Th}$ ) à l'aide d'un spectromètre de masse. La précision est généralement de $2 \sigma$. Cette méthode U/Th atteint ses limites lorsque l'échantillon est contaminé par des éléments détritiques qui apportent aussi du ${ }^{230} \mathrm{Th}$ qui a tendance à vieillir artificiellement l'âge de la stalagmite. Les phénomènes de recristallisation, les porosités, les phases de corrosion-érosion qui peuvent affecter les concrétions perturbent aussi fortement l'enregistrement du signal isotopique de l'uranium. Les phénomènes de corrosion se caractérisent, à l'analyse isotopique, par un faible rapport ${ }^{234} \mathrm{U} /{ }^{238} \mathrm{U}$ en raison du départ préférentiel $\mathrm{du}{ }^{234} \mathrm{U}$ par dissolution, provoquant un rajeunissement artificiel de la concrétion (Maire, 1990). Enfin, la limite de datation de la méthode U/Th se situe à $350-400 \mathrm{ka}$.

Dans notre étude, les deux méthodes ont été utilisées à partir de la stalagmite $\mathrm{BdCl}-19$. Dans un premier temps, extraits à l'aide d'une petite scie circulaire en diamant, six échantillons ont été datés par la méthode U/Th (TIMS) par B. Ghaleb, au Centre de recherche en géochimie et géodynamique (GEOTOP-UQAM-McGill) de l'Université du Québec à Montréal (tab. 2). Les résultats montrent que la stalagmite s'est formée pendant $\sim 6 \mathrm{ka}$. Les âges donnés, non corrigés, sont compris entre $91,7 \mathrm{ka}$ $(+5,5 /-5,2)$ et $97,9 \mathrm{ka}(+2,7 /-2,6)$, avec une précision $\pm 0,5 \%$ (2). Malgré des rapports ${ }^{234} \mathrm{U} /{ }^{238} \mathrm{U}$ cohérents avec ceux obtenus dans d'autres échantillons français et étrangers (Maire, 1990; Audra, 1994; Rihs et al., 1999), les très faibles rapports ${ }^{230} \mathrm{Th} / 232 \mathrm{Th}$, qui indiquent une forte concentration en ${ }^{232} \mathrm{Th}$, en raison d'éléments détritiques venus s'intercaler entre les lamines de calcite, associés aux faibles teneurs en ${ }^{238} \mathrm{U}(28 \mathrm{ppb}$ ), expliquent que les dates fournies n'ont pas pu être considérées comme valables en l'état. La correction du rapport d'activité du ${ }^{230} \mathrm{Th} /{ }^{232} \mathrm{Th}$ de la fraction détritique a révélé un vieillissement artificiel des âges obtenus. Il a donc fallu considérer que l'âge de la stalagmite était probablement beaucoup plus jeune, compris entre les SIM 1 et 5 .

C'est dans cette optique que la méthode du radiocarbone $\left({ }^{14} \mathrm{C}\right)$ a été appliquée, d'abord sur un échantillon sommital de la stalagmite BdCl-19 par D. Genty au Laboratoire des Sciences du Climat et de l'Environnement (LSCE) de Gif-sur-Yvette (fig. 10). Après correction de la proportion de carbone mort établie à $20 \%$, la calibration à l'aide de la courbe de calibration IntCal09 (Reimer et al., 2009) et du logiciel OxCal (https://c14.arch.ox.ac.uk/login/ login.php?Location=/oxcal/OxCal.html) a donné un âge
Fig. 9: Profils géochimiques verticaux réalisés à partir de la microsonde XRF Horiba (lab. CENBG, Bordeaux - Gradignan).

Fig. 9: Vertical geochemical profiles from detrital deposits core infilling the Mud Room, using the Horiba XRF microprobe (lab. CENBG, Bordeaux-Gradignan). 


\begin{tabular}{|c|c|c|c|c|c|c|}
\hline Echantillon & ${ }^{238} \mathrm{U}(\mathrm{ppb})$ & ${ }^{232} \mathrm{Th}(\mathrm{ppb})$ & ${ }^{234} \mathrm{U} /{ }^{238} \mathrm{U}$ & ${ }^{230} \mathrm{Th} /{ }^{234} \mathrm{U}$ & ${ }^{230} \mathrm{Th} /{ }^{232} \mathrm{Th}$ & $\begin{array}{c}\text { Age calculé } \\
(\text { erreur } 2 \sigma)\end{array}$ \\
\hline BdCl-01 & $\begin{array}{c}28.763 \\
( \pm 0.157)\end{array}$ & $\begin{array}{c}11.088 \\
( \pm 0.087)\end{array}$ & $\begin{array}{c}1.0752 \\
( \pm 0.0096)\end{array}$ & $\begin{array}{c}0.5748 \\
( \pm 0.0210)\end{array}$ & $\begin{array}{c}4.899 \\
( \pm 0.180)\end{array}$ & $\begin{array}{c}91774 \\
{[+5,5 /-5,2]}\end{array}$ \\
\hline BdCl-02 & $\begin{array}{c}29.672 \\
( \pm 0.158)\end{array}$ & $\begin{array}{c}13.928 \\
( \pm 0.111)\end{array}$ & $\begin{array}{c}1.0765 \\
( \pm 0.0107)\end{array}$ & $\begin{array}{c}0.5804 \\
( \pm 0.0292)\end{array}$ & $\begin{array}{c}4.068 \\
( \pm 0.204)\end{array}$ & $\begin{array}{c}93150 \\
{[+7,7 /-7,2]}\end{array}$ \\
\hline BdCl-03 & 28.787 & 15.146 & 1.0817 & 0.5906 & 3.710 & 95652 \\
& $( \pm 0.122)$ & $( \pm 0.111)$ & $( \pm 0.0067)$ & $( \pm 0.0245)$ & $( \pm 0.156)$ & {$[+6,6 /-6,2]$} \\
\hline BdCl-04 & 31.336 & 15.679 & 1.0686 & 0.5845 & 3.815 & 94308 \\
& $( \pm 0.131)$ & $( \pm 0.121)$ & $( \pm 0.0076)$ & $( \pm 0.0332)$ & $( \pm 0.217)$ & {$[+8,9 /-8,2]$} \\
\hline BdCl-05 & 28.927 & 12.333 & 1.0831 & 0.5965 & 4.632 \\
& $( \pm 0.176)$ & $( \pm 0.079)$ & $( \pm 0.0091)$ & $( \pm 0.0087)$ & $( \pm 0.068)$ & $\begin{array}{c}97150 \\
{[+2,4 /-2,3]}\end{array}$ \\
\hline BdCl-06 & 28.389 & 22.908 & 1.0756 & 0.5990 & 2.440 \\
& $( \pm 0.154)$ & $( \pm 0.133)$ & $( \pm 0.0062)$ & $( \pm 0.0097)$ & $( \pm 0.042)$ & $\begin{array}{c}97929 \\
{[+2,7 /-2,6]}\end{array}$ \\
\hline
\end{tabular}

Tab. 2: Résultats isotopiques et âges de l'échantillon BdCl-19 par la méthode U/Th.

Tab. 2: Isotopic results and ages of the sample BdCl-19 dated by the U/Th method.

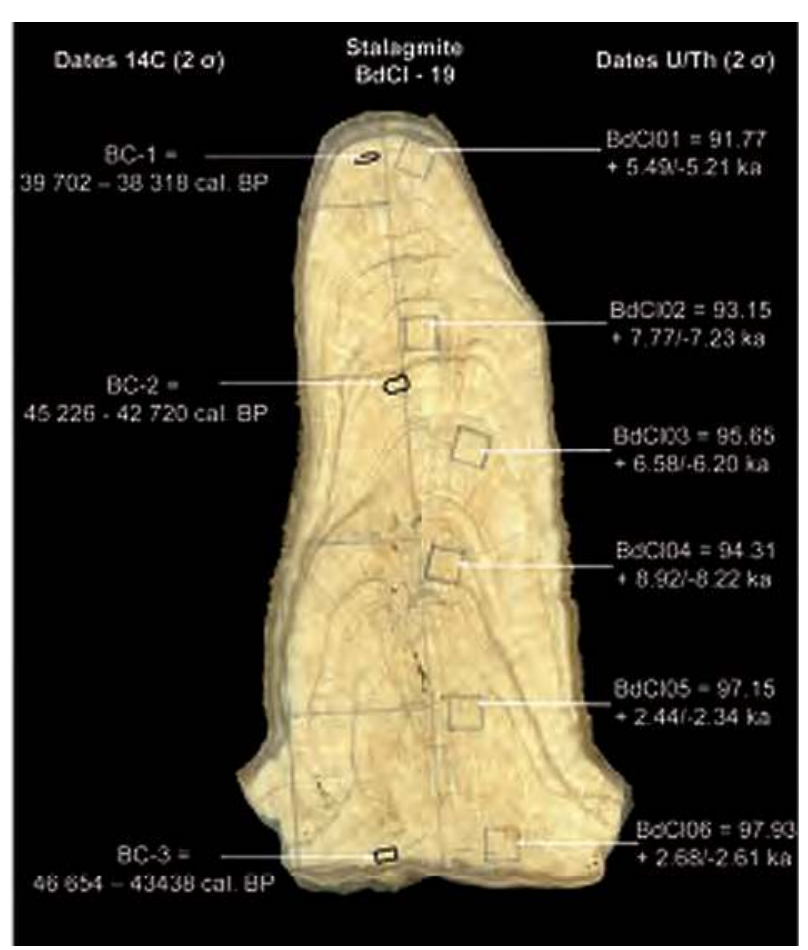

Fig. 10 : Comparaison des datations ${ }^{14} \mathrm{C}$ et $\mathrm{U} / \mathrm{Th}$ sur la stalagmite BdCl-19 qui scelle le remplissage.

La forte proportion de thorium détritique a artificiellement vieilli la concrétion, dont les datations ${ }^{14} \mathrm{C}$ montrent qu'elle s'est formée au cours du SIM 3.

Fig. 10: Comparison of ${ }^{14} \mathrm{C}$ and U/Th datings on the stalagmite BDCL-19 that seals the filling. The high proportion of detrital thorium has aged the speleothem. ${ }^{14} \mathrm{C}$ datings showed it was formed during marine isotope stage 3.

de $39702-38318$ cal. BP (erreur à $2 \sigma$; SacA $15165=$ $34009 \pm 428$ BP). Confortés par ce résultat, deux autres datations radiocarbone ont donné 45226 - 42720 cal. BP (erreur à $2 \sigma$; beta- $286108=42510 \pm 680 \mathrm{BP}$ ) au milieu de la concrétion, et $46654-43438$ cal. BP (erreur à $2 \sigma$; beta- $286109=43500 \pm 680 \mathrm{BP})$ à sa base.

\section{4 - INTERPRÉTATIONS ET DISCUSSION}

La difficulté d'interpréter les résultats vient de trois choses. D'une part, l'absence de datations précises à l'intérieur de la séquence sédimentaire empêche un calage chronologique; d'autre part, l'effet filtre du karst fait que les sédiments piégés dans le karst profond ne sont pas significatifs des conditions paléoclimatiques et paléoenvironnementales dans lesquelles ils se sont déposés, mais de celles dans lesquelles ils se sont formés avant leur sédimentation dans le karst profond. Il y a donc un décalage qu'il est indispensable de prendre en compte. Mais ce décalage est difficile à appréhender au vu du caractère discontinu de la séquence et des nombreux hiatus sédimentaires. Campy (1990) a montré que parfois les lacunes représentent plus de temps écoulé que les phases de sédimentation, ce qui pose un véritable problème d'un point de vue chronostratigraphique.

Cependant, les différentes approches, granulométriques, minéralogiques et géochimiques ont permis d'aboutir à une typologie des dépôts. Trois séquences d'assèchement successives ont été reconnues dans la salle de la Boue établies selon le modèle suivant: d'abord des sables fluviatiles surmontés par des limons argileux homogènes ou lités jusqu'à des faciès typiques de décantation totale. Ce type de séquence sédimentaire a été décrit dans le réseau de Niaux-Lombrives-Sabart dans les Pyrénées ariégeoises (Sorriaux, 1982) et dans la Galerie Aranzadi du réseau de la Pierre Saint-Martin (Maire \& Quinif, 1988). Si Maire et Quinif montrent le lien entre les dépôts de la Galerie Aranzadi et les évolutions climatiques du Pléistocène moyen et supérieur grâce aux nombreuses stalagmites utilisées comme jalons chronologiques par datations U/Th pour positionner les séquences, Sorriaux reste plus prudent quant à l'interprétation paléoclimatique. Comme le note Quinif (1990) : « cette séquence [d'assèchement] n'est pas nécessairement reliée directement à des causes climatiques. En effet, elle traduit le passage du karst d'un système ouvert à un système fermé». La notion d'effet de site est mise au premier plan. Les contraintes géométriques de la salle, la position du remplissage dans le réseau, la position de la nappe phréatique et l'évolution du système karstique ont considérablement joué sur l'enregistrement sédimentaire.

Ainsi, faute de cadre chronologique précis, l'interprétation des résultats ne permet pas d'établir une 
chronostratigraphie ni de reconstituer les contextes paléoenvironnementaux et paléoclimatiques. Toutefois, les résultats obtenus, comparés à des études menées dans des sites voisins, permettent de préciser l'évolution du fonctionnement de la salle de la Boue comme piège sédimentaire.

\section{1 - DATATIONS U/TH ET ${ }^{14} \mathrm{C}$ SUR LA STALAGMITE BDCL-19}

Les résultats ${ }^{14} \mathrm{C}$ confirment une croissance sur $\sim 6 \mathrm{ka}$. En revanche, les âges donnés sont beaucoup plus récents que ceux obtenus par la méthode U/Th et sembleraient confirmer un vieillissement artificiel de la concrétion par un apport de ${ }^{230} \mathrm{Th}$ détritique. Les âges ${ }^{14} \mathrm{C}$, bien qu'ils soient en limite de méthode, sont contemporains du SIM 3. La stalagmite $\mathrm{BdCl}-19$ a très probablement crû pendant cette courte période, entre 45000 et 38000 ans cal. BP, première partie de la seconde moitié de l'interpléniglaciaire weischselien, incluant notamment les événements de Dansgaard/Oeschger (D/O) 12 à 9.

Ainsi, compte tenu du contexte géomorphologique, de la position du dépôt détritique dans la cavité et des âges ${ }^{14} \mathrm{C}$, on peut attribuer le remplissage détritique sous-jacent au SIM 4. Une seconde stalagmite (BdCl-51), située dans le remplissage entre les unités $\mathrm{F}$ et $\mathrm{G}$ a été découverte. Son basculement et les traces de corrosion attestent de son ancienneté. Une seule datation ${ }^{14} \mathrm{C}$ a été effectuée afin de vérifier qu'il ne s'agissait pas d'une concrétion déstabilisée et tombée du sommet du remplissage. La date obtenue non corrigée a donné un âge > 43500 BP (hors limite de la méthode; beta-299501). Elle est donc hors limite de méthode. Une datation U/Th sur cette concrétion devrait permettre de mieux caler la séquence sédimentaire. Mais le problème d'une contamination par du thorium détritique et la très faible teneur en uranium risquent de compromettre le résultat.

\section{2 - DISTRIBUTIONS GRANULOMÉTRIQUES ET DYNAMIQUES HYDRO-SEDIMENTAIRES}

L'analyse granulométrique a permis de distinguer quatre types de sédimentation. Les limons-argileux massifs et homogènes, jaunes, gris et rouges de l'ensemble inférieur sont regroupés autour du type TC sur le diagramme de Passega qui représente les dépôts de talus continental, c'est-à-dire des dépôts de décantation totale. La dynamique hydrologique devait être nulle ou très faible. Ces types dépôts sont liés à des ennoyages prolongés de la salle de la Boue, dans un contexte de lac souterrain lié à des paléo-niveaux de la nappe phréatique de la Touvre. Les fluctuations du niveau de la nappe ont permis la mise en place de dépôts successifs très fins de type limons-argileux, reconnus non seulement dans la salle de la Boue, mais aussi dans la salle des Lacs. L'analyse géochimique a révélé de fortes proportions de $\mathrm{Mn}$ et de Fe qui s'expliquent par des tâches noires et des filaments ferromanganiques. Elle montre que les dépôts de l'ensemble inférieur se sont formés dans un milieu réducteur, fermé et protégé, favorisant une sédi- mentation par décantation totale à partir d'une nappe d'eau permanente qui a connu d'importantes variations de niveau. D'un point de vue minéralogique, ces dépôts présentent les mêmes caractéristiques que ceux étudiés en surface, dont les spectres minéralogiques ont été attribués à la fin du Pliocène (Fridman et al., 1961; Duplaix \& Guillien, 1967; Duplaix, 1970). En effet, les sables de l'unité A et les limons-argileux de l'unité B, C et D montrent un certain nombre de similitudes granulométriques et minéralogiques avec les dépôts du Reuvérien décrit par Fridman et al. (1961), Guillien (1965), Duplaix et Guillien (1967) et Duplaix (1970) dans la vallée de la Tardoire: des sables blancs quartzeux légèrement ferruginisés pour l'unité A, une granulométrie fine montrant une sédimentation dans des eaux très calmes pour les unités $\mathrm{B}, \mathrm{C}$ et $\mathrm{D}$, une très forte proportion de kaolinite et de rares minéraux de socle. On reconnaît pour ces trois dernières unités, l'abondance de zircons, de tourmalines et de disthènes. Ces traits minéralogiques et granulométriques sont ceux du Reuvérien. Cet étage stratigraphique correspond à des dépôts continentaux caractéristiques des ultimes phases chaudes du Pliocène (2,5 - 2,3 Ma). Il a été décrit en France, en Normandie (Clet \& Huault, 1987; Lautridou et al., 1999) et dans le Massif central (Bout, 1967; Pastre et al., 2007) et en Europe du Nord (Zagwijn \& Suc, 1984). En Charente, le Reuvérien a été reconnu en surface comme étant une formation lacustre et fluviatile, s'étendant en amont de La Rochefoucauld jusqu'en aval de Coulgens et de la Bonnieure (Fridman et al., 1961; Guillien, 1965; Duplaix \& Guillien, 1967; Duplaix, 1970). Les formations de la base de la séquence sédimentaire pourraient provenir du remaniement de ces dépôts reuvériens.

Les unités de limons argileux laminés de l'ensemble moyen sont très intéressantes, car elles témoignent d'une modification du régime hydrodynamique dans la cavité. Les dépôts rouges correspondent à des sédiments de suspensions uniformes. Ils traduisent un écoulement à débit très faible précédant un retour aux conditions de décantation totale, dont les limons jaunes sus-jacents sont les témoins. Ils n'ont pas été reconnus dans d'autres galeries de la cavité. Le cortège minéralogique est différent de celui décrit ci-dessus, avec une abondance de hornblende et de grenat (cortège typique de socle), d'oxydes de fer et de quartz altérés. L'origine des sédiments est donc différente par rapport aux unités de l'ensemble inférieur. La présence de quelques quartz rond-mâts ( $<10 \%)$ indique un transport éolien ancien, en provenance du Massif central. De surcroît, la ferruginisation des zones altérées révèle une stabilisation et une pédogenèse de ces dépôts en surface, bien avant leur remobilisation et leur transport jusque dans le karst profond. Enfin, les nombreux impacts et aspérités arrondies qui perforent la surface altérée des quartz sub-anguleux sont le signe d'un transport fluviatile (émoussés-luisants). Ces dépôts pourraient provenir de la couverture cénozoïque d'altérites et de la reprise d'anciennes terrasses plioquaternaires du Bandiat. Leur sédimentation dans le karst souterrain correspond à une décantation d'eau turbide. Elle est associée à des périodes froides enregistrées par la 
minéralogie argileuse de l'unité G. De fait, les rythmites sont souvent interprétées comme la marque de conditions paléoclimatiques alternativement froides / humides et de courtes périodes plus tempérées (Renault, 1967; Maire, 1990; Audra, 1994; Lignier \& Desmet, 2002; Losson et al., 2006). Le système karstique est alors ouvert. Il enregistre les variations sédimentaires d'un paléolac qui ne devait occuper que la salle de la Boue. Ces dépôts, non reconnus dans la salle des Lacs, témoignent de la déconnexion de la salle de la Boue, perchée au-dessus de la salle des Lacs. La salle de la Boue enregistre une sédimentation lente de particules en suspensions diluées, contrôlée par les conditions paléoclimatiques extérieures, ce qui suppose un système karstique ouvert.

Les niveaux sableux associés aux chenaux témoignent aussi des changements des conditions hydrodynamiques. Ils sont d'un grand intérêt, car ils rythment la séquence sédimentaire. Le niveau sableux inférieur (unité A), plus ancien, n'est pas actuellement daté. Il pourrait provenir des dépôts reuvériens décrits ci-dessus. Sans stratification, il témoigne d'une sédimentation à courant fort, typique de crues. Mais son contexte climatique reste encore obscur. Les deux niveaux sableux des ensembles moyen et supérieur (unités $\mathrm{E}$ et I) se situent au niveau des dépôts de suspensions gradées et de roulement. Ils traduisent des mises en charge plus importantes avec des courants forts, creusant des chenaux profonds. Les figures sédimentaires observées dans le niveau sableux intermédiaire (unité E) correspondent à des strates entrecroisées et biseautées. Elles sont liées au comblement progressif du chenal et témoignent de périodes alternatives d'écoulement dynamique de type crue (Cojan \& Renard, 2006). Le niveau sableux de l'ensemble supérieur (unité I) présente un style de stratification différent. Les dépôts se présentent sous la forme d'un litage horizontal sans aucune trace d'érosion, qui témoigne d'un régime d'écoulement moins fort. La bande limono-argileuse rouge à l'intérieur de ce niveau traduit le faible transport de sédiment (granoclassement). Ainsi, même si les unités A, E et I correspondent toutes trois à des dépôts sableux de courant, leurs conditions de sédimentation diffèrent.

Enfin, les dépôts limono-argileux de l'ensemble supérieur (unités J, K et L) sont pour partie regroupés autour du type TC, pour l'autre vers les dépôts de suspensions uniformes. Toutes trois attestent d'un assèchement progressif de la cavité. La mise en place de l'unité J est liée à des processus de ruissellement / colluvionnement qui remobilisent des matériaux antérieurement déposés dans la galerie. De plus, les granules calcaires indiquent des dépôts gravitaires provenant des parois et de la voûte. L'unité $\mathrm{K}$ abonde dans le sens d'une fermeture du système karstique. L'analyse géochimique a permis de mettre en évidence la forte corrélation entre $\mathrm{Sr}$ et $\mathrm{Ca}$ et d'expliquer l'origine authigène du Ca par précipitation du $\mathrm{SrCO}_{3}$. L'analyse minéralogique des argiles a confirmé l'enregistrement de conditions climatiques froides et humides. Une surface d'eau peu profonde alimentée par les infiltrations d'eau s'est progressivement asséchée. L'assèchement de la cavité et l'émersion des dépôts sont enfin attestés par l'unité $\mathrm{L}$, avec une sédimentation de particules mal triées d'origine colluviales, puis par le plancher stalagmitique qui scelle le remplissage.

En résumé, le passage de dépôts successifs limonoargileux de décantation à leur incision par un chenal profond lui-même comblé par des limons puis des sables grossiers fluviatiles révèle un changement style de sédimentation. On peut décrire la séquence suivante: (i) une décantation totale de sédiments limono-argileux, (ii) une phase de chenalisation, (iii) une sédimentation sableuse. Dans les Pyrénées, ce type de séquence a été corrélé à une fonte due à une amélioration climatique (Maire \& Quinif, 1988). Cependant, le contexte morpho-structural charentais est bien différent. Par ailleurs, les nombreux hiatus sédimentaires perturbent considérablement la chronostratigraphie de la séquence du Bois du Clos.

\section{3 - LE PROBLÈME DES HIATUS SÉDIMENTAIRES}

Les nombreuses lacunes sédimentaires complexifient la lecture détaillée de cette séquence. La complexité de la structure d'ensemble et l'hétérogénéité des dépôts marquent une sédimentation polyphasée, entrecoupée de périodes d'émersion et d'érosion.

Quatre grandes discontinuités ont été reconnues. La première correspond à la surface d'érosion qui met en contact l'unité $\mathrm{B}$ et l'unité $\mathrm{C}$. Elle indique un changement des conditions hydro-sédimentaires. Le recreusement a pour origine soit un écoulement libre en chenal soit un soutirage qui suppose un dénoyage total de la cavité. On remarque que le type de sédimentation qui suit est de nouveau marqué par une décantation totale. Aucune sédimentation sableuse n'est venue s'intercaler entre les deux phases de décantation. Le système karstique est donc resté fermé. On ne doit, a priori, cette discontinuité qu'à la très forte amplitude que pouvaient avoir les battements de la nappe. Il est intéressant de signaler qu'aujourd'hui encore les variations piézométriques sont puissantes. En lien avec des variations saisonnières (basses / hautes eaux), elles peuvent atteindre $20 \mathrm{~m}$ au niveau du Bandiat (Larocque, 1997). Ces variations de très grandes amplitudes ont aussi été observées par les spéléologues. Depuis la découverte de la grotte du Bois du Clos en 1990, le niveau des lacs, qui constituent une fenêtre sur l'aquifère, a considérablement varié. En 1991, le niveau est descendu de $7 \mathrm{~m}$.

La deuxième discontinuité est constituée par le profond chenal intermédiaire qui recoupe sur la droite de la coupe les trois unités basales B, C et D. Ce recreusement est associé à une phase de dénoyage en lien avec un abaissement du toit de la nappe aquifère. Des écoulements libres en provenance de l'extérieur ont incisé et évacué une partie des dépôts. Puis un nouveau changement est survenu. La sédimentation est devenue limono-argileuse puis sableuse jusqu' à combler totalement le chenal. Est-ce que ce chenal a une signification locale ou régionale ? Plusieurs études sur des sites archéologiques de grotte charentais révèlent des épisodes de soutirage important. Le site d'Artenac (Delagnes et al., 1999) montre une séquence sédimentaire de plus de $20 \mathrm{~m}$ de hauteur en rive droite de la Bonnieure, présentant à la base la marque de 
puissants soutirages successifs attribués au Pléistocène moyen. Par ailleurs, dans la grotte Bourgeois-Delaunay (la Chaise-de-Vouthon), Debénath (1974) et Bertran (1999) attribuent l'effondrement enregistré à la base de la séquence sédimentaire argileuse au SIM 6, qui survient après l'assèchement et l'émersion des dépôts lacustres dans un contexte climatique très froid (Griggo, 1996). Enfin, deux effondrements majeurs attribués au Pléistocène supérieur ancien ont été reconnus dans la grotte du Placard (Roche, 1965) et dans la grotte de Fontéchevade (Henri-Martin, 1965) à la suite de l'émersion de dépôts argileux. La séquence sédimentaire de la grotte du Bois du Clos pourrait avoir enregistré la transition entre le Pléistocène moyen et le Pléistocène supérieur. Par comparaison avec des études menées sur des sites karstiques proches (Laville et al., 1986), l'étude du remplissage de la salle de la Boue permettrait d'avancer la séquence suivante: (i) décolmatage de la salle de la Boue dû à un soutirage majeur à l'origine du chenal ; (ii) comblement de limons rouges dans le fond du chenal durant sous climat froid et humide (si l'accumulation de fer dans le chenal indique des dépôts de type ferricrète en surface, sa présence dans le karst nous oblige à la considérer comme une période de rhexistasie) ; (iii) accumulation sableuse correspondant à des dépôts de crue; (iv) amélioration des conditions climatiques probablement à l'origine de la formation de spéléothèmes, apparaissant au sommet de l'unité E. Cet enregistrement témoigne également de l'ouverture du système karstique; ouverture qui permettra un retour à une sédimentation détritique sableuse par des écoulements à courant fort (stratification entrecroisée de l'unité E).

La troisième discontinuité se marque par une surface d'érosion majeure qui a presque fait entièrement disparaître l'unité $\mathrm{F}$ et le sommet de l'unité $\mathrm{E}$. Le toit de cette dernière devait arriver au niveau du toit de l'unité D. Au sommet de l'unité E, une stalagmite basculée et corrodée a été découverte. Elle est elle-même en partie recouverte par l'unité F. Cette stalagmite, qui n'a pu être pour le moment datée avec précision, indique en tous les cas une amélioration des conditions climatiques de type tempéré et humide. Recouverte entièrement par l'unité $\mathrm{F}$, elle a été corrodée puis basculée par une séquence d'érosion majeure. L'unité F témoigne d'un ré-ennoiement de la grotte du Bois du Clos, avec des dépôts de décantation d'eau turbide. Le profil géochimique met nettement en évidence de plus fortes proportions de $\mathrm{Rb}$. Les sédiments déposés au-dessus de cette discontinuité ont bien une origine totalement différente de ceux des unités inférieures. Le biseautage de l'unité F (pente à $33^{\circ}$ ) indique une érosion par glissement en masse des sédiments déséquilibrant, basculant la stalagmite érodée et déformant la partie sommitale du remplissage sableux dont les figures de sédimentation (pli de compression) de l'unité E peuvent témoigner. L'origine de cette phase d'érosion ne trouve pas d'échos dans la salle des Lacs, située en contrebas de la salle de la Boue, ni dans les autres grottes de la région. La remobilisation en masse de sédiment peut s'expliquer par des coulées de boue consécutives à des crues (Gillieson, 1986; Bertran, 1999; Ford
\& Williams, 2007). Cette séquence d'érosion est capitale, car elle révèle un glissement en masse dû probablement à un débourrage local. Le soutirage a dû être brutal. Il est lui-même à l'origine d'un effet barrage, bloquant la connexion avec la salle des Lacs. Son influence est nette, car les dépôts sus-jacents présentent tous un pendage sud de $20^{\circ}$ qui contraste avec les dépôts subhorizontaux de l'ensemble inférieur.

La dernière discontinuité met en contact les unités $H$, H', I et J. Faisant suite à une longue séquence de dépôts laminés de décantation, elle se marque par deux choses: un chenal peu profond sur la droite de la coupe; une déformation de la partie sommitale de l'unité I. En effet, l'unité J, constituée de galets mous et plus épaisse sur la partie gauche de la coupe, vient butter contre l'unité I. Les strates horizontales du niveau sableux se déforment progressivement au sommet de la formation. L'origine de cette discontinuité est attribuée à la pression exercée par l'éboulisation gravitaire et le colluvionnement des galets mous. Elle correspond à un processus sédimentaire uniquement local.

\section{4 - COMPARAISON AVEC LES DONNÉES RÉGIO- NALES: MISE EN ÉVIDENCE DES FORÇAGES CLIMATIQUES ET DES EFFETS DE SITE}

L'analyse des variations des dynamiques hydrosédimentaires et des hiatus sédimentaires a révélé que l'ouverture et la fermeture du système karstique apparaissent comme des paramètres primordiaux de contrôle de la sédimentation dans la salle de Boue. La comparaison avec d'autres sites proches a permis de discriminer la part des forçages climatiques externes et celle des paramètres internes. Létude granulométrique, micromorphologique, minéralogique et géochimique a permis de préciser les conditions de transfert sédimentaire dans l'endokarst en discriminant les paramètres locaux et les paramètres régionaux.

Les dynamiques hydrosédimentaires de l'ensemble inférieur correspondent à une remobilisation de dépôts tertiaires mis en place dans un contexte d'ennoiement total de la cavité par décantation due aux battements plus ou moins réguliers des niveaux de la nappe phréatique. Cependant, les sédiments piégés dans le karst profond ne sont pas significatifs des conditions paléoclimatiques et paléoenvironnementales dans lesquelles ils se sont déposés, mais plutôt de celles dans lesquelles ils se sont formés avant leur sédimentation dans le karst profond. Aussi, il semble peu probable que les formations de l'ensemble inférieur se soient mises en place à la fin du Pliocène. En revanche, elles témoignent d'une période de rhexistasie (Erhart, 1956). Les dépôts tertiaires de surface, formés sous des conditions climatiques chaudes et humides, ont été mobilisés à cause d'une déstabilisation morphologique (dégradation climatique, tectonique) qui a favorisé leur piégeage dans le karst profond par des battements réguliers de la nappe aquifère. Nous n'avons actuellement aucun moyen de préciser la période de mise en place de ces dépôts (absence de pollen, aucun reste de grande faune ou de micromammifères). Mais l'hypothèse d'un enregistrement sédimentaire endokarstique d'une 
période alternativement froide / humide, froide / sèche et fraîche / humide du Pléistocène inférieur ou moyen par des formations massives, déposées dans un contexte d'ennoyage total de la cavité et issues du soutirage des dépôts fluviatiles et lacustres de surface, est plausible au vu des résultats granulométriques, minéralogiques et géochimiques. Le Pléistocène inférieur n'est pas bien reconnu en Charente (fig. 11). Les seules traces du Pléistocène moyen ont été reconnues dans la grotte d'Artenac (Delagnes et al., 1999). L'enregistrement sédimentaire par décantation des battements de nappe dans la salle de la Boue et dans la salle des Lacs montre que le système karstique est fermé en amont à tout autre type d'apport sédimentaire. Seule la partie aval du système fonctionne alors par battement de la nappe.

Le hiatus sédimentaire correspondant au chenal de l'ensemble moyen marque probablement l'ouverture du système karstique amont. Le dénoyage de la cavité suivi par un écoulement dynamique (crues) marque un change- ment brutal des conditions hydrodynamiques. Ce décolmatage est à mettre en relation avec une amélioration des conditions climatiques de type tempéré / humide. A l'instar de ce qui se passe alors en Périgord-Quercy (Hoffman, 2005) au Pléistocène moyen, les phases d'encaissement des cours d'eau dans les principales vallées sont presque achevées. Il est possible d'imaginer qu'en Charente, ces phénomènes érosifs se traduisent par des écoulements fluviatiles rapides qui pénètrent dans le karst par des paléopertes débourrées par l'encaissement des cours d'eau. Le puissant soutirage enregistré dans la grotte d'Artenac (Delagnes et al., 1999) témoigne de cette phase et pourrait être corrélé au phénomène de chenalisation enregistré dans la salle de la Boue.

Le retour à une sédimentation détritique fine dans le fond du chenal indique un retour à des conditions climatiques plus froides. Cette période se marque par des oscillations de phases froides / sèches et de phases froides / humides. La longue phase sédimentaire sableuse au-dessus est

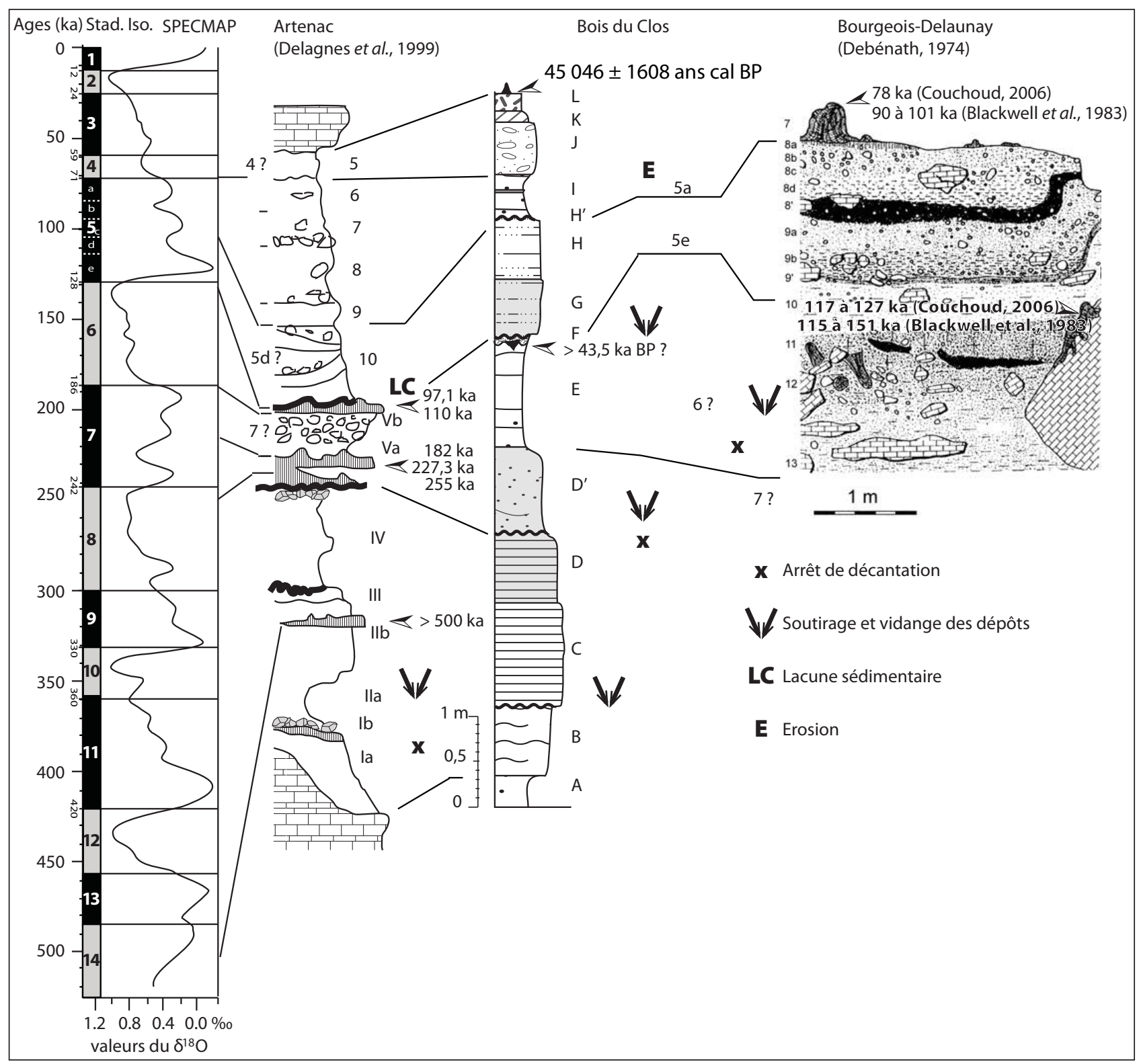

Fig. 11 : Synthèse et comparaison des informations chronostratigraphiques des grottes Bourgeois-Delaunay, d'Artenac et du Bois du Clos. Fig. 11: Synthesis and comparison of chronostratigraphic information from Bourgeois-Delaunay, Artenac and Bois du Clos caves. 
probablement liée à cette période complexe, mais bien enregistrée en Charente (Debénath, 1974; Blackwell et al., 1983 ; Delagnes et al., 1999). L'assèchement de la cavité se marque par la nature limono-argileuse des sédiments et la formation de spéléothèmes au sommet de l'unité E. Elles annoncent une période biostasique caractéristique d'un optimum climatique. La stalagmite du plancher inférieur de la grotte Bourgeois-Delaunay datée du SIM 5e (Debénath et al., 1980; Couchoud, 2006) témoigne de l'amélioration climatique enregistrée en Charente au cours de l'Eemien, confirmée par l'analyse pollinique (Hocine, 1996). La grotte du Bois du Clos pourrait avoir enregistré les variations paléoclimatiques de la fin du Pléistocène moyen et du début du Pléistocène supérieur.

Mais la surface d'érosion majeure qui entaille les unités $\mathrm{F}, \mathrm{D}$ et $\mathrm{E}$ indique un changement brutal des conditions d'enregistrement sédimentaire. Elle apparaît comme le résultat d'un glissement en masse des sédiments, soutirés par un débourrage ponctuel, localisé probablement dans la salle des Lacs. C'est toute la contrainte géométrique de la salle qui s'en trouve modifiée. Désormais les dépôts qui vont s'accumuler dans la salle de la Boue présente un pendage de $20^{\circ}$, en direction d'un point bas situé au sud. Le débourrage soudain a provoqué ipso facto un barrage sédimentaire. Le système karstique se ferme en aval de la salle de la Boue. A partir de ce moment elle est déconnectée de la salle des Lacs. Cette déconnexion se vérifie à la fois par une différence de faciès sédimentaire. Les dépôts des ensembles moyen et supérieur reconnus dans la salle de la Boue n'apparaissent pas dans le remplissage de la salle des Lacs. De surcroît, la courbe du rubidium augmente brusquement à partir de l'unité G. On a une signature géochimique nette du changement de nature et de provenance des sédiments. Associée au potassium, l'augmentation rapide du rubidium dans la séquence sédimentaire montre que les formations moyennes et supérieures sont associées à une sédimentation de type lacustre avec de forts apports détritiques. Cette nouvelle contrainte endokarstique va permettre l'enregistrement sédimentaire de dépôts varvés, contrôlé par les conditions paléoclimatiques extérieures alternativement froides / humides (SIM 5d, 5c et 5b ?). Les oscillations du Pléistocène supérieur tendent progressivement vers une péjoration climatique. Dans les autres sites charentais, ce déséquilibre climatique négatif engendre des phénomènes sédimentaires périglaciaires: démantèlement des parois par gélivation des couches 9 à 5 dans la grotte d'Artenac, gélifraction et coulées de boue révélées par les unités 10 à 8 dans la grotte BourgeoisDelaunay. Les unités $\mathrm{G}$ et $\mathrm{H}$ sont constituées essentiellement de particules fines détritiques ( $\mathrm{Sr}, \mathrm{Rb}, \mathrm{Y}, \mathrm{K}$ et Fe). Dès lors la sédimentation de la salle de la Boue n'est plus contrôlée par les battements de la nappe aquifère, mais par un paléolac souterrain.

L'ensemble supérieur débute par un chenal qui incise peu profondément l'unité H sous-jacente. Elle témoigne d'une nouvelle phase d'assèchement de la cavité en liaison avec une amélioration des conditions climatiques externes (Pujol \& Turon, 1986; SIM 5a?). Le système karstique amont est encore ouvert. L'incision marquée par le chenal révèle des conditions tempérées / humides. Puis le remplissage du chenal par des dépôts sableux indique une nouvelle dégradation climatique (Pujol \& Turon, 1986; SIM 4 ? ou transition SIM 4/3 ?). L'ouverture de la partie amont du réseau est attestée par le piégeage dans la galerie de l'os d'un cheval daté à > 51500 BP (hors limite de la méthode; V. Dujardin, OxA-10421 LYON1375, datation non publiée). Le Bois du Clos fonctionnait alors encore pour quelques temps comme un aven piège à l'instar de la grotte d'Artenac.

Finalement la dernière discontinuité entre l'unité I et J marquerait une fermeture du système karstique aussi à l'amont. La séquence sommitale confirme cette hypothèse. La granulométrie de l'unité J, la composition chimique de l'unité $\mathrm{K}$ et la minéralogie de l'unité L révèlent que l'enregistrement des conditions paléoclimatiques est essentiellement sous la dépendance de facteurs topographiques endokarstiques (sédiments autochtones et parautochtones, Renault, 1967-68; Quinif, 1994; Delannoy, 1997). L'hypothèse plausible est d'y voir une phase froide et humide progressant vers des conditions tempérées (SIM 3) durant laquelle des phénomènes sédimentaires internes (ruissellement, éboulisation gravitaire) dominent. La stalagmite qui scelle le remplissage, datée du SIM 3, confirme la fermeture et l'assèchement de la cavité.

En résumé, bien qu'ayant été largement influencée par les conditions paléoclimatiques et paléoenvironnementales externes, l'analyse combinée granulométrique, minéralogique et géochimique a permis de mettre en évidence la part du site dans les mécanismes hydrosédimentaires: fermeture progressive du réseau, rôle diminuant de la nappe souterraine, déconnexion de la salle de la Boue par rapport à la salle des Lacs en contrebas, géométrie contraignante de la salle de la Boue (débourrages, soutirages, pendants de voûte).

$\mathrm{Au}$ final, un certain nombre d'informations paléoclimatiques ont pu être tirées de l'étude des dépôts. Mais en l'absence de calage chronologique précis il devient difficile de valider ces hypothèses. Par ailleurs, plusieurs processus sédimentaires et d'érosion ont été interprétés comme des phénomènes azonaux de l'endokarst (dépôts de battement de nappe, glissement en masse de sédiments, colluvionnement, ruissellement diffus et éboulisation gravitaire).

\section{5 - CONCLUSION ET PERSPECTIVES}

L'étude morphosédimentaire du remplissage détritique de la grotte du Bois du Clos a permis de mettre en évidence l'influence des différentes contraintes géomorphologiques du site - point bas, cuvettes, barrages constituent autant de pièges sédimentaires - et de la succession des dynamiques hydrologiques - le courant détermine le tri et la distribution des particules - sur l'enregistrement sédimentaire des signaux paléoenvironnementaux et paléoclimatiques. Des études portant sur des sites karstiques de type abri-sous-roche ou porche d'entrée de grottes aboutissent aux mêmes conclusions (Campy, 
1990; Sordoillet, 1990; Ferrier \& Kervazo, 1999). Les lacunes sédimentaires, le caractère discontinu de la sédimentation (Campy, 1990; Quinif, 1990; Ferrier \& Kervazo, 1999) et les effets de barrière, de filtre et de piège (Perroux, 2005; Lans et al., 2006) liés au milieu souterrain, perturbent les signaux associés aux forçages climatiques externes régionaux. Ainsi, bien que les sédiments détritiques du karst profond représentent un fort potentiel d'informations (enregistrement à haute résolution, faible évolution post-dépositionnelle, relative bonne protection des phénomènes érosifs et pédogénétiques), leur étude est limitée par les lacunes sédimentaires et l'absence de datations précises. Ces limites peuvent être dans certains cas palliées grâce aux datations OSL (Optically Stimulated Luminescence) ou par des mesures de nucléides cosmogéniques radioactifs $\left({ }^{10} \mathrm{Be}\right.$ et $\left.{ }^{26} \mathrm{Al}\right)$.

Les changements hydrologiques peuvent être conditionnés par l'évolution particulière du site endokarstique en lui-même (phénomènes de soutirage des points bas, ouverture de pertes en surface liée à des effondrements des galeries supérieures et du toit de la cavité...). Dans le cas présent, les soutirages, ravinements et barrages sédimentaires qui se sont produits dans la salle de la Boue ont contribué à l'isoler de la salle des Lacs dont la sédimentation est essentiellement contrôlée par les battements de la nappe karstique. L'évolution géomorphologique du site a donc conditionné la sédimentation et, de fait, les informations paléo-environnementales archivées.

Les notions d'effet de site et de piège montrent l'importance de croiser les analyses et les angles d'approche. Dans notre étude, l'analyse géochimique a été un apport important. La fluorescence RX a permis de mieux caractériser les dépôts sédimentaires et les conditions de sédimentation en complément de l'approche classique. Même si elle ne permet pas de combler certaines lacunes (datations, liens avec les paléoclimats), elle a permis de mettre en évidence la fin de l'influence du battement de la nappe karstique dans la salle de la Boue grâce à la détection de l'élément $\mathrm{Rb}$. Elle a aussi permis de révéler les conditions très particulières de sédimentation de l'unité $\mathrm{K}$ grâce à la corrélation du $\mathrm{Sr}$ et du $\mathrm{Ca}$. Ainsi, l'analyse géochimique peut apporter une interprétation plus fine que les autres techniques classiques sédimentologiques. Les résultats géochimiques montrent que Ti, $\mathrm{Zr}$, K et Rb sont de bons marqueurs d'apports d'éléments détritiques très fins (bonne corrélation avec la granulométrie et la minéralogie). Enfin, l'anti-corrélation $\mathrm{Fe} / \mathrm{Si}$ est bien corrélée à la granulométrie. Ces résultats sont similaires à d'autres études portant sur des milieux lacustres (Wien et al., 2005; Croudace et al., 2006; Cuven et al., 2010; Kylander et al., 2011). Couplée avec des datations (pollen, diatomées, spéléothèmes...), l'utilisation de la géochimie pourrait devenir un outil d'analyse capital.

Même si les interprétations sont limitées par l'absence de datations, la comparaison avec d'autres sites a permis d'établir des corrélations et a révélé des caractères sédimentologiques et morphologiques communs conférant à certains dépôts une valeur régionale que l'on peut attribuer au climat. La partie étudiée de la séquence de la grotte du Bois du Clos montre un enregistrement sédimentaire discontinu durant une partie du Pléistocène moyen et supérieur. Les SIM 3 et 4 ont été peu reconnus en Charente. La grotte du Bois du Clos pourrait permettre de combler cette lacune régionale. Mais des limites importantes sont à apporter. Par bien des aspects, il semble que la séquence ne soit pas directement reliée à des facteurs climatiques. La signification chronostratigraphique et paléoclimatique des dépôts endokarstiques est réduite. Un certain nombre d'incertitudes demeurent dans notre étude. La datation U/Th de la stalagmite $\mathrm{BdCl}-51$, au milieu de la séquence, pourrait permettre de préciser la chronostratigraphie de la séquence sédimentaire.

\section{REMERCIEMENTS}

Nous tenons à remercier Abderazak El Albani de l'université de Poitiers qui a permis la minéralogie des argiles, Gérard Chabaud du laboratoire EPOC de Bordeaux pour la microgranulométrie, Jean-Christophe Pellegrin de l'université de Bordeaux 3 et Michel Cremer du laboratoire EPOC pour les lames minces. Cet article a aussi bénéficié des corrections fructueuses de Yves Quinif de l'université de Mons en Belgique et de Yves Perrette du laboratoire EDYTEM de Chambéry. Qu'ils en soient ici vivement remerciés.

\section{RÉFÉRENCES BIBLIOGRAPHIQUES}

ANTOINE P., ROUSSEAU D.-D., MOINE O., KUNESCH S., HATTÉ C., LANG A., TISSOUX H., \& ZÖLLER L., 2009 - Rapid and cyclic aeolian deposition during the Last Glacial in European loess: a high-resolution record from Nussloch, Germany. Quaternary Science Reviews, 28 (25/26), 2955-2973.

ATKINSON T.C., HARMON R.S., SMART P.L., \& WALTHAM A.M., 1978 - Paleoclimatic and geomorphic implications of ${ }^{230} \mathrm{Th} /{ }^{234} \mathrm{U}$ dates on speleothems from Britain. Nature, 272 (5648), 24-28.

AUDRA P., 1994 - Karsts alpins. Genèse de grands réseaux souterrains. Exemples: le Tennengebirge (Autriche), l'île de Crémieu, la Chartreuse et le Vercors (France). Karstologia. Mémoires, 17, 1-279.

BASTIN B., 1990 - L'analyse pollinique des concrétions stalagmitiques: méthodologie et résultats en provenance des grottes belges. Karstologia. Mémoires, 2, 3-10.

BELLOT-GURLET L., 1998 - Caractérisation par analyse élémentaire (PIXE et ICP-MS/-AES) d'un verre naturel: l'obsidienne. Application à l'étude de provenance d'objets archéologiques. Thèse de Doctorat, Université Joseph Fourier - Grenoble 1, Grenoble, $298 \mathrm{p}$.

BERTRAN P., 1999 - Dynamique des dépôts de la grotte BourgeoisDelaunay (La Chaise-de-Vouthon, Charente) : apport de la micromorphologie. Paléo, 11, 9-18.

BLACKWELL B., SCHWARCZ H.P., \& DEBÉNATH A., 1983 Absolute dating of hominids and paleolithic artefacts from the cave of La Chaise-de-Vouthon (Charente), France. Journal of Archaeological Science, 10 (6), 493-513.

BLANC J.-J., 2005 - La croissance des spéléothèmes: un enregistrement climatique à haute résolution, analyses d'images, périodes passées et futures, essais d'interprétation. L'Anthropologie, 109 (2), 215-248.

BÖGLI A., 1980 - Karst hydrology and physical speleology. Springer Verlag, Berlin, Heidelberg \& New York, $284 \mathrm{p}$.

BÖNING P., BARD E., \& ROSE J., 2007 - Toward direct, micronscale XRF elemental maps and quantitative profiles of wet marine sediments. Geochemistry Geophysics Geosystems, 8, Q05004, doi:10.1029/2006GC001480.

BOUT P., 1967 - Observations sur le Villafranchien d'Auvergne et du Velay: Compte rendu de l'excursion de l'A.F.E.Q. du 19 au 22 mai 
1966. Bulletin de l'Association Française pour l'Etude du Quaternaire, 4 (1), 3-64.

CAILLEUX A., \& TRICART J., 1959 - Initiation à l'étude des sables et des galets. Volume 1: Texte. Centre de Documentation Universitaire, Paris, $376 \mathrm{p}$.

CAMPY M., 1990 - L'enregistrement du temps et du climat dans les remplissages karstiques : 1'apport de la sédimentologie. Karstologia. Mémoires, 2, 11-22.

CANER L., 2011 - Phyllosilicates des sols: de l'identification à la quantification. Mémoire d'Habilitation à Diriger des Recherches, Université de Poitiers, Poitiers, $146 \mathrm{p}$.

CLET M., \& HUAULT M.-F., 1987 - Les dépôts lagunaires du Reuvérien dans les argiles de la Londe (Normandie, France). Bulletin de l'Association Française pour l'Etude du Quaternaire, 24 (4), 195-202.

COJAN I., \& RENARD M., 2006 - Sédimentologie. Dunod, Paris, $444 \mathrm{p}$

COUCHOUD I., 2006 - Etude pétrographique et isotopique des spéléothèmes du sud-ouest de la France formés en contexte archéologique: contribution à la connaissance des paléoclimats régionaux du stade isotopique 5. Thèse de Doctorat, Université Bordeaux 1, Bordeaux, $323 \mathrm{p}$.

CROUDACE I.W., RINDBY A., \& ROTHWELL R.G., 2006 ITRAX: description and evaluation of a new multi-function X-ray core scanner. In R. Rothwell G. (ed.), New Techniques in Sediment Core Analysis. Geological Society of London, London, 51-63.

CROVISIER J.-L., 1979 - Technique d'induration superficielle pour la réalisation de lames minces dans les matériaux poreux. Cahiers ORSTOM. Pédologie, 17 (3), 229-231.

CUVEN S., 2009 - Validation and use of the micro X-ray fluorescence to characterize clastic varves and paleoclimate quantification of the late Holocene in the Canadian High Arctic. Ph. D. thesis, Universite du Québec, $332 \mathrm{p}$

CUVEN S., FRANCUS P., \& LAMOUREUX S.F., 2010 - Estimation of grain size variability with micro X-ray fluorescence in laminated lacustrine sediments, Cape Bounty, Canadian High Arctic. Journal of Paleolimnology, 44 (3), 803-817.

DANDURAND G., \& MAIRE R., 2011 - Essai de typologie des cavités du karst de La Rochefoucauld (Charente) - Rôle de la «fantômisation » crétacée, du battement de la nappe karstique et d'un effet de site hérité. Travaux du Laboratoire de Géographie Physique Appliquée, 27, 11-28.

DARRÉNOUGUÉ N., DE DECKKER P., FITZSIMMONS K.E. NORMAN M.D., REED L., VAN DER KAARS S., \& FALLON S. 2009 - A late Pleistocene record of aeolian sedimentation in Blanche Cave, Naracoorte, South Australia. Quaternary Science Reviews, 28 (25/26), 2600-2615

DEBARD E., 1997 - Les remplissages karstiques du Bas-Vivarais : karstogenèse, sédimentogenèse et archéologie. Quaternaire, 8 (2/3), 305-317.

DEBENATH A., 1974 - Recherches sur les terrains quaternaires charentais et les industries qui leur sont associées. Thèse d'État, Université de Bordeaux, Bordeaux, 678 p.

DEBÉNATHA., RAYNAL J.-P., \& SCHWARCZ H.P., 1980 - Remarques sur l'identification des planchers stalagmitiques quaternaires. In Groupe d'étude des systèmes carbonatés (ed.), Cristallisation, déformation dissolution des carbonates: Réunion du groupe d'étude des systèmes carbonatés, 17-18 novembre 1980, Bordeaux, Université de Bordeaux 3, Institut de géodynamique. Parrau, Bordeaux, 149-161.

DELAGNES A., TOURNEPICHE J.-F., ARMAND D., DESCLAUX E., DIOT M.-F., FERRIER C., LE FILLÂTRE V., \& VANDER MEERSCH B., 1999 - Le gisement Pléistocène moyen et supérieur d'Artenac (Saint-Mary, Charente) : premier bilan interdisciplinaire. Bulletin de la Société Préhistorique Française, 96 (4), 469-496.

DELANNOY J.-J., 1997 - Recherches géomorphologiques sur les massifs karstiques du Vercors et de la transversale de Ronda (Andalousie) : les apports morphogéniques du karst. Thèse d'État, Université Joseph Fourier - Grenoble I, Grenoble, 678 p.

DELANNOY J.-J., \& CAILLAULT S., 1998 - Les apports de l'endokarst dans la reconstitution morphogénétique d'un karst; exemple de l'Antre de Vénus. Karstologia, 31, 27-41.

DRYSDALE R.N., ZANCHETTA G., HELLSTROM J.C. FALLICK A.E, ZHAO J.X., ISOLA I, \& BRUSCHI G., 2004 Palaeoclimatic implications of the growth history and stable isotope $\left(\delta^{18} \mathrm{O}\right.$ and $\left.\delta^{13} \mathrm{C}\right)$ geochemistry of a Middle to Late Pleistocene stalagmite from central-western Italy. Earth and Planetary Science Letters, 227 (3/4), 215-229.

DUPLAIX S., \& GUILLIEN Y., 1967 - Le plio-quaternaire de la Basse Tardoire (Charente). Bulletin de l'Association Française pour l'Etude du Quaternaire, 4 (2), 171-173.
DUPLAIX S., 1970 - Les minéraux lourds de la Tardoire. Bulletin de l'Association Française pour l'Etude du Quaternaire, 7 (4) 209-227.

DUPLESSY J.-C., LALOU C., DELIBRIAS G., \& NGUYEN H.V., 1972 - Datations et études isotopiques de stalagmites. Applications aux paléotempératures. Annales de Spéléologie, 27 (3), 455-464.

EK C., \& QUINIF Y., 1988 - Les sédiments détritiques de grottes aperçu synthétique. Annales de la Société Géologique de Belgique, 111 (1), 1-7.

EL ALBANI A., MEUNIER A., MACCHIARELLI R., PLOQUIN F., \& TOURNEPICHE J.-F., 2010 - Local environmental changes recorded by clay minerals in a karst deposit during MIS 3 (La Chauverie, SW France). Quaternary International, 241 (1/2), 26-34.

ERHART H., 1956 - La genèse des sols en tant que phénomène géologique. Esquisse d'une théorie géologique et géochimique, biostasie et rhexistasie. Masson, Paris, $90 \mathrm{p}$.

FAIRCHILD I.J., FRISIA S., BORSATO A., \& TOOTH A.F., 2007 Speleothems. In D.J. Nash \& S.J. McLaren (eds.), Geochemical Sediments and Landscapes. Blackwell, Oxford, 200-245.

FAIRCHILD I.J., \& TREBLE P.C., 2009 - Trace elements in speleothems as recorders of environmental change. Quaternary Science Reviews, 28 (5/6), 449-468.

FERRIER C., 1994 - Le contexte environnemental du peuplement paléolithique de Bulgarie du Nord. Le karst de Karlukovo et ses dépôts. Thèse de Doctorat, Université Bordeaux 1, Bordeaux, $451 \mathrm{p}$.

FERRIER C., \& KERVAZO B., 1999 - Réflexions sur la variabilité de 1'enregistrement sédimentaire en entrée de grotte. Etudes de Géographie Physique, 28 Suppl., 89-94.

FRIDMAN R., TERS M, \& GUILLIEN Y., 1961 - Minéraux lourds de la Basse Tardoire et de la Charente moyenne. Bulletin de la Société Préhistorique Française, 58 (11), 763-772.

FORBES M.S., \& BESTLAND E.A., 2007 - Origin of the sedimentary deposits of the Naracoorte Caves, South Australia. Geomorphology, $86(3 / 4), 369-392$

FORD D., \& WILLIAMS P., 2007 - Karst Hydrogeology and Geomorphology. John Wiley \& Sons, Chichester, $562 \mathrm{p}$

GAINE M., 1973 - Une méthode d'induration des échantillons du sol à l'aide de vernis. Cahiers - ORSTOM. Pédologie, 11, 265-267.

GASCOYNE M., 1992 - Palaeoclimate determination from cave calcite deposits. Quaternary Science Reviews, 11 (6), 609-632.

GENTY D., \& DEFLANDRE G., 1998 - Drip flow variations under a stalactite of the Père Noël cave (Belgium). Evidence of seasonal variations and air pressure constraints. Journal of Hydrology, 211 $(1 / 4), 208-232$

GENTY D., MASSAULT M., GILMOUR M., BAKER A., VERHEYDEN S., \& KEPENS E., 1999 - Calculation of past dea carbon proportion and variability by the comparison of $\mathrm{AMS}{ }^{14} \mathrm{C}$ and TIMS U/Th ages on two Holocene stalagmites. Radiocarbon, 41 (3), 251-270

GENTY D., BAKER A., \& VOKAL B., 2001 - Intra- and inter-annual growth rate of modern stalagmites. Chemical Geology, $176(1 / 4)$ 191-212.

GENTY D., 2002 - Spéléothèmes et paléoenvironements. In J.-C. Miskovsky (ed.), Géologie de la Préhistoire: méthodes, techniques, applications. Association pour l'étude de l'environnement géologique de la préhistoire, Paris, 1345-1362

GENTY D., BLAMART D., OUAHDI R., GILMOUR M., BAKER A., JOUZEL J., \& VAN-EXTER S., 2003 - Precise dating of Dansgaar-Oeschger climate oscillations in western Europe from stalagmite data. Nature, 421 (6925), 833-837.

GILLIESON D., 1986 - Cave sedimentation in the New Guinea Highlands. Earth Surface Processes and Landforms, 11 (5), 533-543.

GRIGGO C., 1996 - Etablissement de courbes climatiques quantifiées à partir des communautés animales pléistocènes suivi d'une application aux gisements de l'Abri Suard (Charente) et la grotte de BoisRagot (Vienne). Paléo, 8, 81-97.

GUILLIEN Y., 1965 - La Basse Tardoire. Formations lacustres, formations fluviales et paléosols du Reuvérien à l'Holocène. Bulletin de l'Association Française pour l'Etude du Quaternaire, 2 (3), 205-210.

HÄUSELMANN P., 2004 - Les grottes de Saint-Béat. Formation, historique, exploration. Speleo Projects, Allschwil, $256 \mathrm{p}$

HENRI-MARTIN G., 1965 - La grotte de Fontéchevade. Bulletin de l'Association Française pour l'Etude du Quaternaire, 2 (3) 211-216.

HERAUD-PIÑA M.A., 1996 - Le karst du Yucatan. Pays des Mayas Presses Universitaires de Bordeaux, Bordeaux, $282 \mathrm{p}$

HOCINE F., 1996 - Etude palynologique de l'abri paléolithique Bourgeois-Delaunay (La Chaise, Charente). Quaternaire, 7 (4), 187-196. 
HOFFMANN F., 2005 - Les tufs et travertins en Périgord-Quercy. Karstologia. Mémoires, 13, 1-260.

HOLTZAPFFEL T., 1985 - Les minéraux argileux. Préparation. Analyse diffractométrique et détermination. Publication - Société géologique du Nord, 12, 1-136.

JEANNIN P.-Y., KLOETZLI U., \& LALOU J.-C., 1990 - Remplissages karstiques et paléoclimats. Karstologia. Mémoires, 2, 1-64.

KATSUTA N., TAKANO M., KAWAKAMI S., TOGAMI S., FUKUSAWA H., KUMAZAWA M., \& YASUDA Y., 2007 - Advanced micro-XRF method to separate sedimentary rhythms and event layers in sediments: its application to lacustrine sediment from Lake Suigestu, Japan. Journal of Paleolimnology, 37 (2), 259-271.

KIDO Y., KOSHIKAWA T., \& TADA R., 2006 - Rapid and quantitative major element analysis method for wet fine-grained sediments using an XRF microscanner. Marine Geology, 229 (3/4), 209-225.

KOSHIKAWA T., KIDO Y., \& TADA R., 2003 - High-resolution rapid elemental analysis using an XRF microscanner. Journal of Sedimentary Research, 3 (5), 824-829.

KYLANDER M.E., AMPEL L., WOHLFARTH B., \& VERES D., 2011 - High-resolution X-ray fluorescence core scanning analysis of Les Echets (France) sedimentary sequence: new insights from chemical proxies. Journal of Quaternary Science, 26 (1), 109-117.

LANS B., MAIRE R., ORTEGA R., DEVES G., BACQUART T., PLAISIR C., QUINIF Y., \& PERRETTE Y., 2006 - Les stalagmites du réseau du trou Noir (Gironde) : rôle de l'effet de site dans l'enregistrement du signal climatique et environnemental. Karstologia, 48, 1-22.

LAROCQUE M., 1997 - Intégration d'approches quantitatives de caractérisation et de simulation des aquifères calcaires fissurés. Application à l'aquifère karstique de La Rochefoucauld (Charente, France). Thèse de Doctorat, Université de Poitiers, Poitiers, 247 p.

LAUTRIDOU J.-P., BAIZE S., CLET M., COUTARD J.-P., \& OZOUF J.-C., 1999 - Les séquences plio-pléistocènes littorales et estuariennes de Normandie (France). Quaternaire, $10(2 / 3)$, 161-169.

LAVILLE H., RAYNAL J.-P., \& TEXIER J.-P., 1986 - Le dernier interglaciaire et le cycle climatique würmien dans le sud-ouest et e Massif central français. Bulletin de l'Association Française pour l'Etude du Quaternaire, 23 (1/2), 35-46.

LE FILLÂTRE V., 2001 - Les dépôts dans le karst en Périgord-Quercy et leurs significations. Thèse de Doctorat, Université Bordeaux 3 , $497 \mathrm{p}$.

LIGNIER V., \& DESMET M., 2002 - Les archives sédimentaires quaternaires de la grotte sous les Sangles (Bas-Bugey, Jura méridional, France). Indices paléo-climatiques et sismo-tectoniques. Karstologia, 39, 27-46.

LOSSON B., 2003 - Karstification et capture de la Moselle (Lorraine, France): vers une identification des interactions. Thèse de Doctorat, Université de Metz, 3 volumes, 825 p.

LOSSON B., CORBONNOIS J.,ARGANT J., BRULHET J., PONSBRANCHU E., \& QUINIFY., 2006 - Interprétation paléoclimatique des remplissages endokarstiques de la vallée de la Moselle à Pierrela-Treiche (Lorraine, France). Géomorphologie: Relief, Processus, Environnement, 2006 (1), 37-48

MAIRE R., \& QUINIF Y., 1988 - Chronostratigraphie et évolution sédimentaire en milieu alpin dans la galerie Aranzadi (Gouffre de la Pierre Saint-Martin, Pyrénées, France). Annales de la Société Géologique de Belgique, 111 (1), 61-77.

MAIRE R., 1990 - La haute montagne calcaire. Karsts, cavités, remplissages, Quaternaire, paléoclimats. Karstologia. Mémoires, 3, 1-731.

MCDERMOTT F., 2004 - Palaeo-climate recontruction from stable isotope variations in speleothems: a review. Quaternary Science Reviews, 23 (7/8), 901-918.

PASSEGA R., 1964 - Grain size representation by C.M. patterns as a geological tool. Journal of Sedimentary Research, 34 (4), 830-847.

PASTRE J.-F., LAGEAT Y., CANTAGREL J.-M., \& DEFIVE E., 2007 - Morphogenèse plio-quaternaire et repères volcaniques: l'exemple de la vallée de l'Allier (Massif central, France). In M.-F André (ed.), Du continent au bassin versant. Théories et pratiques en géographie physique (Hommage au Professeur Alain Godard). Presses Universitaires Blaise-Pascal, Clermont-Ferrand, 543-558.

PERRETTE Y., 1999 - Les stalagmites : archives environnementales et climatiques à haute résolution. Présentation des protocoles d'étude et premiers résultats sur des spéléothèmes du Vercors. Karstologia, 34, 23-44.

PERROUXA.-S., 2005 - Les remplissages détritiques endokarstiques. Contribution méthodologique à la lecture des mémoires paléogéographiques et environnementales. Application aux systèmes karstiques de Choranche (Vercors) et d'Orgnac (Bas-Vivarais). Thèse de Doctorat, Université de Savoie, Chambéry, 418 p.
PETERSON L.C., HAUG G.H., HUGHEN K.A., \& RÖHL U., 2000 Rapid Changes in the Hydrologic Cycle of the Tropical Atlantic During the Last Glacial. Science, 290 (5498), 1947-1951.

POMEL S., \& MAIRE R., 1995 - Relations entre les remplissages souterrains et la déstabilisation de l'environnement. Karstologia. Mémoires, 6, 101-120.

POMEL S., \& MAIRE R., 1997 - Exemple d'enregistrement des changements climatiques et de l'anthropisation dans les remplissages endokarstiques de Chine centrale (Hubei). Quaternaire, 8 (2/3), 119-128.

PUJOL C., \& TURON J.-L., 1986 - Comparaison des cycles climatiques en domaine marin et continental entre 130000 et 28000 ans B.P. dans l'hémisphère Nord. Bulletin de l'Association Française pour l'Etude du Quaternaire, 1 (2), 17-25.

QUINIF Y., DUPUIS C., BASTIN B., \& JUVIGNÉ E., 1979 - Étude d'une coupe dans les sédiments quaternaires de la grotte de la Vilaine Source (Arbre, Belgique). Annales de la Société Géologique de Belgique, 102 (1), 229-241.

QUINIF Y., 1990 - La datation des spéléothèmes (U/Th) appliquée aux séquences sédimentaires souterraines pour une mise en évidence des ruptures paléoclimatiques. Karstologia. Mémoires, 2, 23-32.

QUINIF Y., 1994 - Les dépôts endokarstiques. Concepts et méthodologies. In R. Maire, S. Pomel \& J.-N. Salomon (eds.), Enregistreurs et indicateurs de l'évolution de l'environnement en zone tropicale. Presses Universitaires de Bordeaux, Talence, 55-72.

QUINIFY., MÉON H., \& YANS J., 2006 - Nature and dating of karstic filling in the Hainaut Province (Belgium). Karstic, geodynamic and paleogeographic implications. Geodinamica Acta, 19 (2), 73-85.

REIMER P.J, BAILLIE M.G.L, BARD E., BAYLISS A., BECK J.W, BLACKWELL P.G, RAMSEY C.B., BUCK C.E., BURR G.S., EDWARDS R.L., FRIEDRICH M., GROOTES P.M., GUILDERSON T.P., HAJDAS I., HEATON T.J., HOGG A.G., HUGHEN K.A., KAISER K.F., KROMER B., MCCORMAC F.G., MANNING S.W., REIMER R.W., RICHARDS D.A., SOUTHON J.R., TALAMO S., TURNEY C.S.M., VAN DER PLICHT J., \& WEYHENMEYE C.E., 2009 - Intcal09 and Marine09 radiocarbon age calibration curves, 0-50,000 Years Cal Bp. Radiocarbon, 51 (4), $1111-1150$

RENAULT P., 1967-68 - Contribution à l'étude des actions mécaniques et sédimentologiques dans la spéléogénèse. Annales de Spéléologie, 22 (1), 5-267; 23 (1), 259-307; 23 (3), 529-596.

RENAULT P., 1990 - Réflexions sur la notion de remplissages karstiques. Karstologia. Mémoires, 2, 61-62.

RIHS S., POIDEVIN J.-L., \& CONDOMINES M., 1999 - Premiers âges U/Th sur la grotte des Demoiselles (Hérault) : évolution karstique et relation paléoclimatique. Quaternaire, 10 (4), 293-297.

ROCHE J., 1965 - La grotte du Placard. Bulletin de l'Association Française pour l'Etude du Quaternaire, 2 (3), 245-250.

ROUILLER, 1987 - Etude des systèmes karstiques de la Touvre et de la Lèche (Angoulême, Charente). Géologie, hydrodynamique, hydrochimie. Thèse de Doctorat, Université d'Avignon et des Pays de Vaucluse, $220 \mathrm{p}$.

SORDOILLET D., 1999 - Un exemple d'enregistrement anthropique et climatique: le remplissage de la grotte du Gardon (Ambérieu-enBugey, Ain, France). Etudes de Géographie Physique, 28 suppl., 179-184.

SORRIAUX P., 1982 - Contribution à l'étude de la sédimentation en milieu karstique Le système de Niaux-Lombrives-Sabart (Pyrénées ariégeoises). Thèse de Doctorat, Université Paul Sabatier - Toulouse, Toulouse, $255 \mathrm{p}$

VAKS A., BAR-MATTHEWS M., AYALON A., SCHILMAN B., GILMOUR M., HAWKESWORTH C.J., FRUMKIN A., KAUFMAN A., \& MATTHEWS A., 2003 - Paleoclimate reconstruction based on the timing of speleothem growth and oxygen and carbon isotope composition in a cave located in the rain shadow in Israel. Quaternary Research, 59 (2), 182-193.

VELDE B., \& MEUNIER A., 2008 - The origin of clay minerals in soils and weathered rocks. Berlin \& London, $406 \mathrm{p}$.

WHITE W.B., 2007 - Cave sediments and paleoclimate. Journal of Cave and Karst Studies, 69 (1), 76-93.

WIEN K., KÖLLING M., \& SCHULZ H.D., 2005 - Close correlation between $\mathrm{Sr} / \mathrm{Ca}$ ratios on bulk sediments from the southern Cape Basin and the SPECMAP record. Geo-Marine Letters, 25 (4), 265-271.

WILSON M.J., 1999 - The origin and formation of clay minerals in soils: Past, present and future perspectives. Clay Minerals, 34 (1), 7-25.

ZAGWIJN W.H., \& SUC J.-P., 1984 - Palynostratigraphie du PlioPléistocène d'Europe et de Méditerranée nord-occidentale: corrélations chronostratigraphiques, histoire de la végétation et du climat. Paléobiologie Continentale, 14 (2), 475-483. 